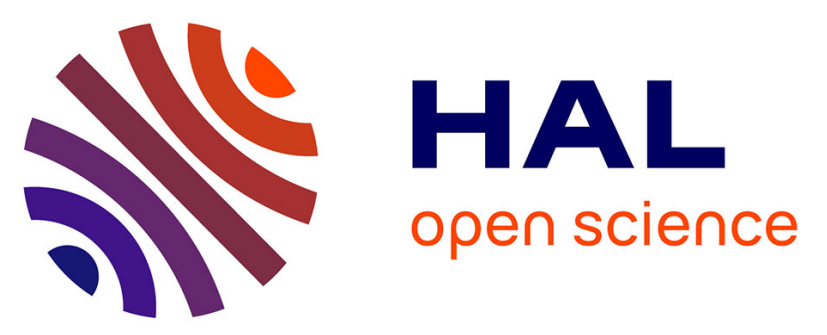

\title{
Ultrafast Oxidation of a Tyrosine by Proton-Coupled Electron Transfer Promotes Light Activation of an Animal-like Cryptochrome
}

Fabien Lacombat, Agathe Espagne, Nadia Dozova, Pascal Plaza, Pavel Müller, Klaus Brettel, Sophie Franz-Badur, Lars-Oliver Essen

\section{To cite this version:}

Fabien Lacombat, Agathe Espagne, Nadia Dozova, Pascal Plaza, Pavel Müller, et al.. Ultrafast Oxidation of a Tyrosine by Proton-Coupled Electron Transfer Promotes Light Activation of an Animallike Cryptochrome. Journal of the American Chemical Society, 2019, 141 (34), pp.13394-13409. 10.1021/jacs.9b03680 . hal-02284305

\section{HAL Id: hal-02284305 https://hal.science/hal-02284305}

Submitted on 5 Nov 2019

HAL is a multi-disciplinary open access archive for the deposit and dissemination of scientific research documents, whether they are published or not. The documents may come from teaching and research institutions in France or abroad, or from public or private research centers.
L'archive ouverte pluridisciplinaire HAL, est destinée au dépôt et à la diffusion de documents scientifiques de niveau recherche, publiés ou non, émanant des établissements d'enseignement et de recherche français ou étrangers, des laboratoires publics ou privés. 


\section{Article}

\section{Ultrafast Oxidation of a Tyrosine by Proton-Coupled Electron Transfer Promotes Light Activation of an Animal-like Cryptochrome}

Fabien Lacombat, Agathe Espagne, nadia dozova, Pascal Plaza, Pavel Müller, Klaus Brettel, Sophie Franz-Badur, and Lars-Oliver Essen

J. Am. Chem. Soc., Just Accepted Manuscript • DOI: 10.1021/jacs.9b03680 • Publication Date (Web): 01 Aug 2019

Downloaded from pubs.acs.org on August 5, 2019

\section{Just Accepted}

"Just Accepted" manuscripts have been peer-reviewed and accepted for publication. They are posted online prior to technical editing, formatting for publication and author proofing. The American Chemical Society provides "Just Accepted" as a service to the research community to expedite the dissemination of scientific material as soon as possible after acceptance. "Just Accepted" manuscripts appear in full in PDF format accompanied by an HTML abstract. "Just Accepted" manuscripts have been fully peer reviewed, but should not be considered the official version of record. They are citable by the Digital Object Identifier (DOI@). "Just Accepted" is an optional service offered to authors. Therefore, the "Just Accepted" Web site may not include all articles that will be published in the journal. After a manuscript is technically edited and formatted, it will be removed from the "Just Accepted" Web site and published as an ASAP article. Note that technical editing may introduce minor changes to the manuscript text and/or graphics which could affect content, and all legal disclaimers and ethical guidelines that apply to the journal pertain. ACS cannot be held responsible for errors or consequences arising from the use of information contained in these "Just Accepted" manuscripts. 


\title{
Ultrafast Oxidation of a Tyrosine by Proton-Coupled Electron Transfer Promotes Light Activation of an Animal-like Cryptochrome
}

\author{
Fabien Lacombat, ${ }^{a}$ Agathe Espagne, ${ }^{a}$ Nadia Dozova, ${ }^{a}$ Pascal Plaza, ${ }^{*, a}$ Pavel Müller, ${ }^{*, b}$ Klaus Brettel, ${ }^{b}$ \\ Sophie Franz-Badur, ${ }^{c}$ Lars-Oliver Essen*,c \\ a PASTEUR, Département de chimie, École normale supérieure, PSL University, Sorbonne Université, CNRS, 75005 \\ Paris, France. E-mail: pascal.plaza@ens.fr \\ b Institute for Integrative Biology of the Cell (I2BC), CEA, CNRS, Univ. Paris-Sud, Université Paris-Saclay, 91198, Gif- \\ sur-Yvette cedex, France. E-mail: pavel.muller@i2bc.paris-saclay.fr \\ c Department of Chemistry, LOEWE Center for Synthetic Microbiology, Philipps University, 35032 Marburg, \\ Germany. E-mail: essen@chemie.uni-marburg.de
}

\begin{abstract}
The animal-like cryptochrome of Chlamydomonas reinhardtii (CraCRY) is a recently discovered photoreceptor that controls the transcriptional profile and sexual life cycle of this alga by both blue and red light. CraCRY has the uncommon feature of efficient formation and longevity of the semi-reduced neutral form of its FAD cofactor upon blue light illumination. Tyrosine $\mathrm{Y}_{373}$ plays hereby a crucial role by elongating as fourth member the electron transfer (ET) chain that comprises the tryptophan triad otherwise found in most other cryptochromes and DNA photolyases. Here, we report the full mechanism of light-induced FADH• formation in CraCRY using transient absorption spectroscopy from hundreds of femtoseconds to seconds. Electron transfer starts from ultrafast reduction of excited FAD to $F A D^{\bullet-}$ by the proximal tryptophan $(0.4 \mathrm{ps})$ and is followed by delocalized migration of the produced $\mathrm{WH}^{\bullet+}$ radical along the tryptophan triad (3.7 and $55 \mathrm{ps}$ ). Oxidation of $\mathrm{Y}_{373}$ by coupled ET to $\mathrm{WH}^{\bullet+}$ and deprotonation then proceeds in $\sim 800 \mathrm{ps}$, without any significant kinetic isotope effect, nor a $\mathrm{pH}$ effect between $\mathrm{pH} 6.5$ and 9.0. The $\mathrm{FAD}^{\bullet-} / \mathrm{Y}_{373} \bullet$ pair is formed with high quantum yield ( $60 \%$ ); its intrinsic decay by recombination is slow ( $50 \mathrm{~ms})$, favoring reduction of $\mathrm{Y}_{373}$ by extrinsic agents and protonation of $F A D^{\bullet-}$ to form the long-lived, red-light absorbing $\mathrm{FADH} \bullet$ species. Possible mechanisms of tyrosine oxidation by ultrafast proton-coupled ET in CraCRY, a process about 40 times faster than the archetypal tyrosine-Z oxidation in photosystem II, are discussed in detail.
\end{abstract}




\section{INTRODUCTION}

Cryptochrome blue-light receptors are responsible for various biological photoinduced responses, such as plant photomorphogenesis, circadian rhythm entrainment in animals or presumably magnetoreception in migrating birds. ${ }^{1-4}$ The triggering mechanism of cryptochromes is based on the photoreduction of their flavin cofactor (FAD: flavin adenine dinucleotide), in a very similar way as in the so-called photoactivation reaction of photolyases, a group of closely related enzymes able to photorepair UV-damaged DNA. ${ }^{1,5-8}$ Some bifunctional cryptochromes even act both as light receptors and DNA-repairing enzymes. ${ }^{9-14}$ The evolutionary links between the different members of the photolyase and cryptochrome superfamily (PCSf) may be found in various reports..$^{15-19}$

A new type of cryptochrome was recently discovered in the green alga Chlamydomonas reinhardtii, exhibiting homology to animal but not plant cryptochromes, called animal-like cryptochrome (the C. reinhardtii protein is noted CraCRY). In contrast to other cryptochromes, CraCRY appears to be capable of inducing a genetic response not only to blue, but also to red light. ${ }^{20-22}$ This feature is probably related to another unique property of CraCRY, the very efficient formation and stabilization of its semireduced (red light-absorbing) flavin cofactor $\left(\mathrm{FADH}^{\bullet}\right)$ that can be easily accumulated under blue light illumination and lives for seconds to minutes (depending on $\mathrm{pH}$ ). ${ }^{20,23}$ This process relies on the oxidation of the phenolic moiety of the tyrosine residue $Y_{373}$ as its mutation to phenylalanine abolishes this effect. ${ }^{24} Y_{373}$ is located at the distal end of the "canonical" tryptophan triad $\left(W_{399}, W_{376}\right.$ and $W_{322}$ in CraCRY; see Figure 1A) that is conserved in most members of the PCSf -except the subfamilies of bacterial (6-4) photolyases and class II photolyases - and is involved in the photoreduction of FAD. 3,7,25 In the canonical PCSf members, the excited flavin abstracts an electron from a nearby (proximal) tryptophan, which gets in turn reduced by a second (medial) tryptophan, before being reduced by a third (distal) one. The distal tryptophan is usually surface-exposed and can hence be reduced by external electron donors such as thiol compounds ${ }^{26}$ or $\mathrm{NAD}(\mathrm{P}) \mathrm{H} .{ }^{27,28}$ For animal-like cryptochromes and (6-4) photolyases, a fourth aromatic residue, $Y_{373}$ in CraCRY and $W_{360}$ in the (6-4) photolyase from Xenopus laevis, has been found to act as final electron donor of an extended electron transfer chain. ${ }^{24,29-32}$

Transient absorption spectroscopy (TAS) has been extensively used to study the mechanistic details of the photoreduction of photolyases ${ }^{31,33-40}$ and cryptochromes, taking advantage of the rather distinct absorption spectra of the species involved (Figure 1B). ${ }^{35,39,41}$ Ultrafast TAS studies have in particular shown that when the flavin is initially oxidized $\left(F A D_{o x}\right)$, the primary electron transfer $(E T)$ takes place in less than one picosecond, ${ }^{31,35,37,39,41}$ producing a radical pair of reduced flavin $\left(F A D^{\bullet-}\right.$ ) and oxidized 


\footnotetext{
${ }^{+}$The notation $\mathrm{WH}^{\bullet+}$ indicates the protonated tryptophanyl radical, while $\mathrm{W}^{\bullet}$ refers to the deprotonated radical. $\ddagger \mathrm{Y} \bullet$ stands for the deprotonated tyrosyl radical; the corresponding protonated species is noted $\mathrm{YH}^{\bullet+}$.
} 
radical is strongly acidic (pKa $\sim-2$ ), ${ }^{60}$ so when ET is coupled to deprotonation of $\mathrm{YH}^{\bullet+}$, the overall reaction is well downhill, i.e. exergonic.

Another case of light-induced tyrosine oxidation was more recently discovered in BLUF (blue-light sensing using FAD) photoreceptor proteins. ${ }^{61-63}$ In the dark-adapted SIr 1694 BLUF, ultrafast (tens of picoseconds) ET from $\mathrm{Y}_{8}$ to the photoexcited FAD cofactor and similarly fast PT from $\mathrm{Y}_{8} \mathrm{H}^{\bullet+}$ to $F A D^{\bullet-}$ via a glutamine residue yield an $\mathrm{Y}_{8}{ }^{\bullet} / \mathrm{FADH}^{\bullet}$ radical pair. ${ }^{61,62}$ This pair recombines on a 100 -ps timescale, but rearrangements of the hydrogen bond network (and a related red shift of the FAD absorption spectrum) persist for seconds and represent the signaling state of the photoreceptor. In the light-adapted SIr 1694 BLUF, formation the of $\mathrm{Y}_{8} \bullet / \mathrm{FADH}^{\bullet}$ pair is even faster ( $\left.1 \mathrm{ps}\right)$, by concerted proton-coupled electron transfer. ${ }^{63}$

Proton-coupled electron transfer (PCET) has been the subject of intense theoretical work and experimental studies in chemistry and biology because of its crucial role in avoiding high energy intermediates in chemical and enzymatic catalysis, notably for solar energy conversion.64-69 As summarized by Hammes-Schiffer and Stuchebrukhov, ${ }^{64}$ two types of coupled reactions can be distinguished. In concerted electron and proton transfer (PT) reactions, both ET and PT occur in one step. ${ }^{63}$ In sequential reactions, the transitions occur in two steps: ET followed by PT (noted ET/PT) or PT followed by ET (PT/ET). In both cases, the first step is typically uphill in energy and the second step is downhill to the final product state. Tyrosine oxidation in the BLUF domain may constitute an exception, which is commented further at the end of Section 4.2.2. A hallmark of a concerted PCET reaction is the observation of a substantial normal kinetic isotope effect (KIE), i.e. the reaction is slowed down when the transferred proton is replaced by a deuteron. Sequential ET/PT and PT/ET typically show a (normal) KIE only when PT becomes rate limiting.

The absence of a KIE on the oxidation of tyrosine in PSII in $~ 30$ ns may point to a sequential PT/ET, where the hydroxyl proton of the tyrosine moves to a nearby histidine residue prior to rate-limiting ET. ${ }^{50,53}$ A slower (few $\mu$ s) tyrosine oxidation in PSIl depleted of the oxygen-evolving complex, however, showed a normal KIE and was hence assigned to concerted PCET. ${ }^{49}$

The presumed oxidant of tyrosine in CraCRY, namely $\mathrm{W}_{322} \mathrm{H}^{\bullet+}$, is likely to have an even lower reduction potential (1.07 $\mathrm{V}$ for the $\mathrm{WH}^{\bullet+} / \mathrm{WH}$ couple in aqueous solution ${ }^{54}$ ) than $\mathrm{P} 60^{+}$in PSII, suggesting that tyrosine oxidation in CraCRY occurs by some type of PCET as well. The main objective of the present work was to resolve the kinetics of tyrosine oxidation in CraCRY and to contribute to establishing its reaction mechanism. Ultrafast pump-probe and real-time transient absorption spectroscopy on timescales from hundreds of $\mathrm{fs}$ to hundreds of $\mathrm{ms}$ were used to monitor the complete reaction 
sequence of flavin photoreduction. Wild-type (WT) CraCRY was compared to its Y373F mutant. ${ }^{\S}$ This mutation was expected to ablate the electron donor character of $Y_{373}$ and enable us to distinguish reactions involving $\mathrm{Y}_{373}$ from preceding reactions that should not be significantly affected by the mutation.

\section{MATERIALS AND METHODS}

\subsection{Sample preparation}

The Chlamydomonas reinhardtii animal-like cryptochrome (CraCRY) samples were prepared as recently described. ${ }^{70}$

For transient absorption experiments, we used a $50 \mathrm{mM}$ phosphate buffer at pH 8.4, containing 100 $\mathrm{mM} \mathrm{NaCl}$ and $20 \%$ glycerol. The $\mathrm{D}_{2} \mathrm{O}$ buffer was composed of heavy water $(99.9$ atom $\% \mathrm{D}$, SigmaAldrich) and $\mathrm{D}_{2} \mathrm{O}$-exchanged glycerol $\left(10 \% \mathrm{v} / \mathrm{v}\right.$ glycerol in $\mathrm{D}_{2} \mathrm{O}$, dried in a rotary evaporator; procedure repeated twice). Some samples had to be concentrated with centrifugal concentrators (Vivaspin 500, Sartorius or Amicon Ultra-4, Sigma-Aldrich; molecular weight cutoff: $30 \mathrm{kDa}$ ) to achieve suitable optical densities. Prior to the experiments, CraCRY solutions were passed through a gel-filtration column (Micro Bio-Spin6, BioRad), equilibrated with the working buffer, to eliminate unbound FAD. The steady-state absorption spectra of CraCRY samples used for femtosecond transient absorption spectroscopy are shown in SI, Section S1 (Figure S1).

\subsection{Femtosecond Transient Absorption Spectroscopy (TAS)}

\subsubsection{Setup}

Polarized transient absorption anisotropy was here used to both access the transient population dynamics, through the isotropic transient absorption, and probe orientation of the transient species with the associated transient absorption anisotropy. Anisotropy is indeed particularly useful to help distinguishing the different $\mathrm{WH}^{\bullet+}$ radicals involved in the photoreduction process, as was previously done for other photolyases and cryptochromes.31,40,41,71 The pump-probe setup, with white-light continuum probe, has been previously described. $31,39,40$ The pump beam was tuned at $475 \mathrm{~nm}$. By comparison with a reference sample of tris-(2,2'-bipyridyl)-ruthenium(II) complex, ${ }^{72^{* *}}$ it was checked

\footnotetext{
$\S$ The abridged notations WT and Y373Y will be used to refer to these variants.

${ }^{* *}$ Systematic measurements on the $\left[\mathrm{Ru}(\mathrm{bpy})_{3}\right]^{2+}$ complex were also used to (i) ensure that no major alteration of the transient spectra occurred upon varying the pump-probe delay over the 3.2-ns range of an optical delay line,
} 
that the fraction of excited molecules in the probed volume was about $3 \%$ (case of WT in $\mathrm{H}_{2} \mathrm{O}$ buffer), which lies in the linear regime.

In order to avoid probing the same region consecutively, the sample cuvette (1-mm optical path) was continuously moved back and forth in horizontal and vertical directions, each pump shot irradiating an area of the order of $0.01 \mathrm{~mm}^{2}$. A relatively large volume ( $250 \mu \mathrm{L}$ in a 10-mm wide cell) of sample was used for the experiment with WT-CraCRY in $\mathrm{H}_{2} \mathrm{O}$ buffer. Alternatively, four smaller cells (2-mm wide, containing $\sim 60 \mu \mathrm{L}$ ) were used for WT in $\mathrm{D}_{2} \mathrm{O}$ buffer, each one being irradiated for a shorter time while the others were recovering in the dark. One $60-\mu \mathrm{L}$ cell was sufficient for Y373F since FADH ${ }^{\bullet}$ does not accumulate so easily in this CraCRY mutant. ${ }^{24,29}$ Steady-state absorption spectra of the samples were regularly recorded during the experiments to check that no significant accumulation of $\mathrm{FADH}^{\bullet}$ or sample degradation occurred. All experiments were performed at room temperature $\left(20^{\circ} \mathrm{C}\right)$.

\subsubsection{Data analysis}

The polarized differential absorption spectra $\left(\Delta A_{\|}\right.$and $\left.\Delta A_{\perp}\right)$, obtained from the transmitted probe beam intensities, were first corrected from the chirp of the probe beam, which was independently measured by recording cross-phase modulation ${ }^{73}(X P M)$ in the pure solvent. These raw polarized spectra were transformed into isotropic transient absorption spectra $\left(\Delta A_{\text {iso }}\right)$ and transient anisotropy spectra $(r)$ using Equations 1 and 2. Scattering of the pump beam was masked in the spectra, both for data analysis and figure presentation.

$$
\left\{\begin{array}{l}
\Delta A_{\text {iso }}=\frac{1}{3}\left(\Delta A_{\|}+2 \Delta A_{\perp}\right) \\
r=\frac{\Delta A_{\|}-\Delta A_{\perp}}{\Delta A_{\|}+2 \Delta A_{\perp}}
\end{array}\right.
$$

Notably, the transient absorption spectra of WT samples displayed a weak signal at negative time delays. This signal is due to a long-lived photoproduct $\left(F A D^{\bullet-} / Y^{\bullet}\right.$ pair $^{29}$ ) produced by the preceding pump pulse ( $3 \mathrm{~ms}$ before the probe only shot and $6 \mathrm{~ms}$ before pump and probe shot), but still detectable because the 2D movement imposed on the sample cell was not fast enough to regenerate entirely the probed sample volume between two probe pulses. This spurious signal has been averaged, smoothed, and subtracted from the raw transient absorption data. The resulting spectra are presented here.

Multiexponential global analysis was performed simultaneously on the parallel and perpendicular data as previously described. ${ }^{31,39,40}$ Effective fits of the isotropic and anisotropy data were derived from

and (ii) make experiments recorded on different days spectrally comparable (minor corrections based on the $\left[\mathrm{Ru}(\mathrm{bpy})_{3}\right]^{2+}$ complex spectra were applied). 
the root fits with Equations 1 and 2. The time constants of the fits will be presented in Table 1 and the corresponding isotropic decay-associated spectra (DADS) provided for reference in SI, Section 4.4 (Figure S10). The global fits are however here more conveniently summarized by the evolution-associated difference spectra (EADS), which correspond to a virtual cascading model with unit quantum yield between state $i$ and state $i+1$ and increasing lifetimes. ${ }^{74}$ The polarized EADS were in turn converted into isotropic EADS and evolution-associated anisotropy spectra (EAAS) using Equations 1 and 2.

Spectral analysis of some isotropic EADS were performed as previously described. ${ }^{40}$ The aim is to fit the spectra by a Beer-Lambert sum of contributions from different species $\left(F A D_{o x}, F A D^{\bullet-}, W H^{\bullet+}, Y^{\bullet}\right)$, the spectra of which are taken from literature (see Figure 1B and SI, Section S5.1). To improve the quality of the fits, the reference spectra were modified with two intertwined parameters, a shift $s$ and a scaling factor $u$, so that spectrum $\varepsilon(\lambda)$ becomes $\varepsilon^{\prime}(\lambda)=\varepsilon(u(\lambda-s))$. To avoid ambiguities coming from the overlap of reference spectra, fits were preferred where the number of species in the sum could be kept low. In particular, we focused on the difference between consecutive spectra, as follows: EADS $_{i+1}$ was fitted with $\mathrm{EADS}_{i}$ as a first fit component, multiplied by an adjustable factor $(\phi)$ understood as the yield of the $i \rightarrow i+1$ phase, plus additional components reflecting composition changes taking place during this step.

\subsection{Real-time TAS}

Transient absorption kinetics on nanosecond to millisecond timescales were measured on two different setups described in detail elsewhere. ${ }^{42,75,76}$ In experiments on the sub-ns/ns timescales, the samples were excited at $355 \mathrm{~nm}$ by a Nd:YAG laser (Continuum Leopard SS-10, pulse duration of $100 \mathrm{ps,}$ repetition rate $2 \mathrm{~Hz}$, and pulse energy in the order of $\sim 2 \mathrm{~mJ}$ per $\mathrm{cm}^{2}$ ). In all other experiments, the samples were excited at $475 \mathrm{~nm}$ by a Nd:YAG-pumped optical parametric oscillator (OPO; Brilliant B/Rainbow, Quantel, France, pulse duration of $5 \mathrm{~ns}$, repetition rate $2 \mathrm{~Hz}$ or single flashes, and energy in the order of $\sim 5 \mathrm{~mJ}$ per $\mathrm{cm}^{2}$ ). Laser energies were estimated from the amplitudes of signals obtained with the $\left[\mathrm{Ru}(\mathrm{bpy})_{3}\right] \mathrm{Cl}_{2}$ actinometer ${ }^{77}$ under otherwise identical conditions and geometry.

Monitoring light was provided by continuous-wave lasers listed previously. ${ }^{76} 2 \times 2 \times 10 \mathrm{~mm}$ cells were used (excitation pulses entered the sample through the $2 \times 10 \mathrm{~mm}$ window; monitoring light through the $2 \times 2 \mathrm{~mm}$ window). The monitoring light beams were attenuated by neutral density filters and, for the experiments on ns to $\mu$ s timescales, were mechanically chopped to produce rectangular light pulses of $140 \mu$ s duration and energy in the order of $1 \mu \mathrm{J}$ at the entrance of the cell, thus avoiding significant 
actinic effects. These pulses were synchronized with the excitation laser flash (see Ref. ${ }^{75}$ for more details).

Signals on the sub-ns/ns timescales were recorded using the Alphalas UPD-200-UP photodiode (rise time $<175 \mathrm{ps}$; sensitive area $0.1 \mathrm{~mm}^{2}$; bandwidth $2 \mathrm{GHz}$ ), connected directly to the Infiniium $81004 \mathrm{~B}$ digital oscilloscope (from Agilent; bandwidth $10 \mathrm{GHz}$ ). Signals on the ns/ $\mu \mathrm{s}$ timescales were recorded using the Alphalas UPD-500-UP photodiode (rise time $<500$ ps; sensitive area $0.5 \mathrm{~mm}^{2}$; bandwidth 600 $\mathrm{MHz}$ ), connected via the Femto HCA electronic signal amplifier (DC-325 MHz, $28 \mathrm{~dB}$ ) to a the Tektronix DSA602A digital oscilloscope with bandwidth limit set to $100 \mathrm{MHz}$. Signals on the $\mathrm{ms} / \mathrm{s}$ timescales were amplified by the AM502 differential amplifier (Tektronix) with a bandwidth limited to $100 \mathrm{kHz}$.

All experiments were carried out at room temperature $\left(\sim 25^{\circ} \mathrm{C}\right)$ and the concentration of both WT and Y373F proteins in most experiments was $\sim 60 \mu \mathrm{M}$. Only for experiments on the ns timescale, a higher concentrated $(\sim 110 \mu \mathrm{M})$ WT sample was used in order to improve the signal-to-noise ratio (at all wavelengths except $457 \mathrm{~nm}$ ).

\section{RESULTS}

\subsection{Femtosecond TAS}

\subsubsection{Isotropic spectral dynamics}

The transient absorption spectra of WT-CraCRY, both WT and Y373F mutant, recorded between $300 \mathrm{fs}$ and $3.2 \mathrm{~ns}$ after excitation at $475 \mathrm{~nm}$, are presented in Figure S4 of SI, Section S3.1, together with a detailed description of them. In brief, at early pump-probe delays of (e.g. $300 \mathrm{fs}$ ), one recognizes the typical features of excited $\mathrm{FAD}_{\mathrm{ox}}\left(\mathrm{FAD}_{\mathrm{ox}}{ }^{*}\right)$, with a bleaching band at $447 \mathrm{~nm}$, stimulated emission (SE) at $538 \mathrm{~nm}$ and excited-state absorption (ESA) bands around 366, $507 \mathrm{~nm}$ and over $570 \mathrm{~nm}$. The following evolution may approximately be divided into three phases. From 0.3 to 2 ps (Figure S4A), the SE band decays and is substituted by a broad positive band, peaking around $600 \mathrm{~nm}$. The changes in this phase resemble closely those observed in several oxidized PCSf members and attributed to primary charge separation forming a FAD ${ }^{\bullet-} \mathrm{WH}^{\bullet+}$ pair. ${ }^{31,39-41}$ Between $\sim 2$ and $\sim 230$ ps (Figure S4B), all bands decay partially without much change of their shape, indicating some loss of $F A D^{\bullet-} / \mathrm{WH}^{\bullet+}$ pairs by charge recombination. Between 230 and 3200 ps (Figure S4C), all bands go on decaying partially but one observes that the transient absorption band at around $600 \mathrm{~nm}$ decays to much greater extent and becomes increasingly flatter, consistent with data obtained by real-time TAS and tentatively assigned to 
reduction of $\mathrm{WH}^{\bullet+}$ by $\mathrm{Y}_{373}$ (see Section 3.2). The evolution of the transient absorption spectra of WTCraCRY measured in deuterated buffer is essentially identical (SI, Section S3.2).

The Y373F mutant (Figure 2D-F) behaves very similarly to the WT as far as the first phase is concerned but exhibits substantial differences afterwards. Firstly, the amplitude of the decay during the second phase is much smaller than for WT and, secondly, the third phase is almost absent; only a very weak decay of all bands without shape alteration occurs between 80 ps and $3.2 \mathrm{~ns}$.

\subsubsection{Kinetic analysis}

All polarized transient absorption spectra of CraCRY could be globally fitted with a sum of 4 exponential components and a plateau. Table 1 summarizes the obtained time constants (with errors of fit corresponding to a confidence interval of 95\%). An illustration of the quality of such fits may be found in SI, Section S4.1, with selected kinetic traces of both isotropic transient absorption and transient anisotropy of WT in $\mathrm{H}_{2} \mathrm{O}$.

Figure $2(A, C)$ shows the isotropic EADS of WT and Y373F in $\mathrm{H}_{2} \mathrm{O}$ buffer (for WT in $\mathrm{D}_{2} \mathrm{O}$ see $\mathrm{SI}$, Section S4.2). The evolution phases qualitatively sketched in Section 3.1.1 are here more precisely defined. The initial excited-state decay occurs in about 0.4 ps for both WT and Y373F. The second phase is divided into two kinetic steps, characterized by time constants of 3.7 and 55 ps for WT (4.9 and 56 ps in $\left.\mathrm{D}_{2} \mathrm{O}\right)$, and 3.4 and 41 ps for Y373F. These time constants are similar in all cases but the amplitude associated to the 41-ps decay of Y373F is, in relative value, smaller than that of the 55-ps decay of WT (Figure $2 \mathrm{~A}, \mathrm{C})$. As mentioned above, the fourth decay of WT in $710 \mathrm{ps}$, with conspicuous flattening of the $600 \mathrm{~nm}$ band and decay below $350 \mathrm{~nm}$, is almost absent in Y373F. One observes instead a very small homothetic decrease of all bands, in 630 ps. Figure 3 displays the EADS2-5 of WT and Y373F ( $\mathrm{H}_{2} \mathrm{O}$ buffer), normalized at the maximum of the bleaching band (NEADS). This normalization approximately compensates for the transient absorption losses due to the recovery of the initial state by charge recombination. Figure 59 (SI, Section S4.3) presents the same data in one-to-one comparison of the NEADS of WT and Y373F. The effect of the Y373F mutation is clearly seen in those figures to block the prominent spectral changes (decay of the absorption bands around $600 \mathrm{~nm}$ and $340 \mathrm{~nm}$, relative absorption increase in the 390430-nm range), observed in WT during the EADS4 $\rightarrow$ EADS5 kinetic step. Minor changes consisting essentially of a small decay of the broad band around $600 \mathrm{~nm}$ observed during the EADS3 $\rightarrow$ EADS4 step in the WT are also blocked by the $Y_{373}$ mutation. 


\subsubsection{Transient anisotropy}

The raw transient anisotropy spectra are provided for reference in SI, Section S3. The evolutionassociated anisotropy spectra (EAAS) deduced from the global analysis procedure are shown in Figure 2 (B, D) for WT in $\mathrm{H}_{2} \mathrm{O}$ and in Figure S8B for WT in $\mathrm{D}_{2} \mathrm{O}$ buffer. In brief, the four kinetic phases described above can also be clearly distinguished according to their anisotropy. The initial phase is characterized by very large changes between 500 and $650 \mathrm{~nm}$, due to the decay of SE. Subsequently, and starting with the simpler case of Y373F, one observes marked changes between 330 and $375 \mathrm{~nm}$ as well as between ca. 510 and $650 \mathrm{~nm}$, during the $2^{\text {nd }}$ and $3^{\text {rd }}$ steps. The last kinetic step is accompanied by minimal alterations of the anisotropy. In the case of WT, the anisotropy changes during the $2^{\text {nd }}$ step are similar to those of Y373F but differences start to appear during the $3^{\text {rd }}$ step; The marked decay around $510 \mathrm{~nm}$ in Y373F is in particular significantly smaller in WT. During the last kinetic step, an increase is observed for WT at this wavelength while no change occurs in Y373F. The final anisotropy spectrum of WT becomes very noisy above $\sim 700 \mathrm{~nm}$ and below $350 \mathrm{~nm}$ because the corresponding isotropic spectrum (denominator in Equation 2) approaches here zero value. These regions have been masked in Figure 2 $(B, D)$.

\subsection{Real-time TAS}

\subsubsection{Sub-nanosecond to nanosecond timescale}

To monitor the photoreactions of CraCRY on the sub-ns to ns timescale, we used a sub-ns TAS setup (see Section 2.3) with excitation pulses at $355 \mathrm{~nm}$ (i.e. in the near UV absorption band of $F A D_{o x}$ ). Signals recorded at representative wavelengths for WT CraCRY and its Y373F mutant are shown in Figure 4A and $4 \mathrm{~B}$, respectively (for other wavelengths, see Figures S2A and S3A). $405 \mathrm{~nm}$ in particular lies close to the expected absorption maximum of the $\mathrm{Y}$ radical (see Figure 1B); note that a strong absorption increase due to $F A D$ reduction from $F A D_{o x}$ to $F A D^{\bullet-}$ is also expected at this wavelength. $457 \mathrm{~nm}$ is close to the absorption maximum of $\mathrm{FAD}_{\mathrm{ox}}$ in the blue in order to monitor the negative absorption changes (bleaching) due to the reduction of $\mathrm{FAD}_{\mathrm{ox}}$ to $\mathrm{FAD}^{\bullet-}$; note that tyrosine and tryptophan radicals exhibit very weak absorptions at this wavelength so their contributions to the signal are expected to be minor compared to those associated with the redox state changes of FAD. At $488 \mathrm{~nm}$, the neutral $\mathrm{W}^{\bullet}$ typically absorbs much stronger $(\sim 2.5 \times)$ than the protonated $\mathrm{WH}^{\bullet+}$ (see Figure $\left.1 \mathrm{~B}\right)$. And finally at $562 \mathrm{~nm}$, where essentially only the tryptophan radicals contribute, the opposite is the case - the molar absorption coefficient of the $\mathrm{WH}^{\bullet+}$ radical is much higher $(\sim 3 \times)$ than that of the $\mathrm{W}^{\bullet}$ radical (see Figure $1 \mathrm{~B}$ ). 
For the Y373F mutant (Figure 4B), the high-time-resolution signals show essentially only a very slight decline (that will be resolved on a longer timescale, see Section 3.2.2). For the WT, however, one observes prominent kinetic features already on the ns timescale (Figure 4A). In particular, at $562 \mathrm{~nm}$ (red trace in Figure 4A), the signal exhibits a major decay from an initial level comparable to that in the Y373F mutant (when corrected for concentration difference) to $20 \%$ of its initial amplitude within a few nanoseconds. Similar signals were observed at 515, 594 and $639 \mathrm{~nm}$ (Figure S2A), strongly suggesting that they represent disappearance of a $\mathrm{WH}^{\bullet+}$ radical (see the absorption spectrum of $\mathrm{WH}^{\bullet+}$ in Figure 1B). A global fit of all signals (at all measured wavelengths) with two shared time constants (using Equation S1) yielded $\tau_{1}=550 \mathrm{ps}$ and $\tau_{2}=5.6 \mathrm{~ns}$ at an amplitude ratio of approx. 3:1 for signals at $515 \mathrm{~nm}$ and above (see Figure S2B). The 550 ps phase may contain contributions from even faster processes that could not be resolved at the instrumental time resolution of 300 ps (see above for data obtained by ultrafast spectroscopy).

A priori, disappearance of the $\mathrm{WH}^{\bullet+}$ signature in WT CraCRY could be not only caused by the expected $E T$ from $\mathrm{Y}_{373}$ but also due to back ET from $\mathrm{FAD}^{\bullet-}$ or to deprotonation. Deprotonation of $\mathrm{WH}^{\bullet+}$ would form the neutral radical $\mathrm{W}^{\bullet}$ and should be accompanied by an absorption increase at $488 \mathrm{~nm}$ (as observed in the Y373F mutant; see Figure S3B). Instead, the disappearance of $\mathrm{WH}^{\bullet+}$ within a few nanoseconds in WT CraCRY was accompanied by bleaching at $488 \mathrm{~nm}$ (green trace in Figure 4A), excluding deprotonation as major process. Back ET from $\mathrm{FAD}^{\bullet-}$ to $\mathrm{WH}^{\bullet+}$ should be accompanied by recovery of the bleaching of the absorption band of $\mathrm{FAD}_{\mathrm{ox}}$ around $450 \mathrm{~nm}$. A partial recovery in approx. $1 \mathrm{~ns}$ was indeed observed at $457 \mathrm{~nm}$ (blue trace in Figure 4A), but represents only 20\% of the initial bleaching and can hence only account for $\sim 20 \%$ of the disappearance of $\mathrm{WH}^{\bullet+}$.

ET from $\mathrm{Y}_{373}$ is hence the most plausible major cause of disappearance of $\mathrm{WH}^{\bullet+}$ within a few nanoseconds. For energetic reasons outlined in the Introduction, this ET step is likely to be coupled to deprotonation of $\mathrm{Y}_{373}$ (PCET) and should be accompanied by an absorption increase around $405 \mathrm{~nm}$ due to formation of a $Y^{\bullet}$ radical (see solution spectrum in Figure $1 \mathrm{~B}$ ) that is superimposed to the strong absorption increase due to the preceding formation of FAD ${ }^{\bullet-}$ (Figure 1B). The WT signal at $405 \mathrm{~nm}$ (black trace in Figure 4A) shows a minor (10-15\%) absorption increase with kinetics consistent with the slower phase $(5.6 \mathrm{~ns})$ of disappearance of $\mathrm{WH}^{\bullet+}$ observed at $\lambda>500 \mathrm{~nm}$. Within the first $1 \mathrm{~ns}$ after excitation, there was, however, a slight absorption decrease at $405 \mathrm{~nm}$. This is likely to be the result of an overcompensation of a rise by partial decay of the strong absorption of $\mathrm{FAD}^{\bullet-}$ due to its recombination with $\mathrm{WH}^{\bullet+}$ (see Discussion). 
Because of the likely involvement of tyrosine deprotonation, we followed the kinetics of $\mathrm{WH}^{\bullet+}$ in WT CraCRY also at other $\mathrm{pH}$ values. Figure $\mathrm{S} 2 \mathrm{C}$ shows the corresponding $562 \mathrm{~nm}$ signals at pH 6.5, 7.5, 8.4 (the "standard" pH for all other experiments shown here) and 9.0. All signals exhibit essentially the same decay kinetics and also the same amplitudes of the decay (see SI, Section S2.2 for further details).

\subsubsection{Nanosecond to second timescale}

To complete our observations on the ns to $s$ timescale, we used a TAS setup with $\sim 5$ ns time resolution (see Section 2.3), with excitation pulses tuned at $475 \mathrm{~nm}$. Figure 5 compares transient absorption kinetics in the WT protein and in the Y373F mutant at three representative monitoring wavelengths (see Figures S2 and S3 for additional wavelengths). Note that absolute signal amplitudes are not directly comparable to those of the sub-ns setup due to different excitation conditions and different protein concentrations.

Upon flash excitation, the signals recorded for the WT CraCRY up to $70 \mu$ s (Figure 5A, 5B) exhibit step-like absorption changes, containing a very small and unresolved fast kinetic phase in $\leq 10 \mathrm{~ns}$ (minor growth at $405 \mathrm{~nm}$, minor decay at $562 \mathrm{~nm}$ and essentially no visible change at $457 \mathrm{~nm}$ ). All signals remain essentially constant within the time frames of Figure $5 \mathrm{~A}$ and $5 \mathrm{~B}$ and decay only on a much longer timescale (in $\sim 50 \mathrm{~ms}$, see inset of Figure 5B). The amplitudes of the $562 \mathrm{~nm}$ signals amount to mere $\sim 10 \%$ of the amplitudes of the $457 \mathrm{~nm}$ signals (in absolute values) in WT CraCRY, indicating that there are virtually no tryptophan radicals (protonated or deprotonated) left when the initial fast kinetic steps are over. The remaining small positive signal at $562 \mathrm{~nm}$ can be attributed to the red tail in the absorption spectrum of the $\mathrm{FAD}^{\bullet-}$ radical (Figure $1 \mathrm{~B}$ ).

In the case of the Y373F mutant (Figure $5 \mathrm{C}$ and D), all signals exhibit a biphasic and complete decay $\left(\tau_{1} \sim 260 \mathrm{~ns}, \tau_{2} \sim 10 \mu \mathrm{s}\right)$. At the beginning of the time window of Figure $5 \mathrm{C}$, the amplitude of the signal at $562 \mathrm{~nm}$ amounts to $\sim 60 \%$ of the signal at $457 \mathrm{~nm}$ and even after the first $260 \mathrm{~ns}$ phase, this number still remains as high as $\sim 45 \%$ (Figure $5 \mathrm{C}$ and $\mathrm{D}$ ). This indicates that tryptophan radicals are present until the decay is completed (in $\sim 70 \mu \mathrm{s}$ ). In most members of the PCSf that contain the canonical tryptophan triad, the distal $\left(3^{\text {rd }}\right) \mathrm{WH}^{\bullet+}$ radical deprotonates in several hundreds of nanoseconds, ${ }^{33,42}$ in competition with recombination with FAD ${ }^{\bullet-}{ }^{42}$ This also seems to be the case for the Y373F mutant of CraCRY: While the 260 ns phase represents $\sim 30 \%$ of the signal decay at 405 and $457 \mathrm{~nm}$ (essentially due to FAD•-/WH ${ }^{\bullet+}$ recombination), it represents $\sim 50 \%$ at $562 \mathrm{~nm}$ (and even more at higher wavelengths) where both recombination and deprotonation of $\mathrm{WH}^{\bullet+}$ contribute (Figs. 6A and S3). The intrinsic time constants for $\mathrm{WH}^{\bullet+}$ deprotonation and $\mathrm{FAD}^{\bullet-} / \mathrm{WH}^{\bullet+}$ recombination derived from these data are $\sim 400 \mathrm{~ns}$ and $\sim 850$ 


\section{DISCUSSION}

Our experimental results are discussed below in chronological order of the photoinduced events during the light activation of CraCRY, focusing, however, on kinetics and mechanism of fast and efficient tyrosine oxidation that has no precedent in other members of the PCSf and is highly relevant for the understanding of proton coupled electron transfer (Section 4.2). A tentative reaction scheme is provided to facilitate the discussion (Scheme 1).

\subsection{Reactions preceding tyrosine oxidation}

\subsection{1. $\quad$ Nature and kinetics of the photoproducts}

Starting with the simpler case of Y373F, we find that the isotropic EADS (Figure 2C) are reminiscent of those previously reported for other members of the $\operatorname{PCS}^{31,39,40}$ and, more specifically, highly similar to those of the W370F mutant of the 6-4 photolyase from Xenopus laevis (X/64). ${ }^{31}$ In the latter mutant, the aromatic tetrad serving as ET chain in the WT is analogously reduced to the classical tryptophan triad, as in the Y373F mutant of CraCRY. Accordingly, EADS1 can be straightforwardly assigned to $\mathrm{FAD}_{\text {ox }}{ }^{*}$ and EADS2 to the $\mathrm{FAD}^{\bullet-} / \mathrm{WH}^{\bullet+}$ radical pair formed by photoinduced ET from the proximal tryptophan ( $\mathrm{W}_{399}$ in $\mathrm{CraCRY}$ ) to $\mathrm{FAD}_{\mathrm{ox}}{ }^{*}$ in 0.43 ps. EADS3-5 reflect $\mathrm{FAD}{ }^{\bullet-} / \mathrm{WH}^{\bullet+}$ states, in which the oxidation hole has migrated along the tryptophan triad (in competition with charge recombination), or relaxed states. In the present case, the initial photoreduction of the flavin produces a particular spectrum (EADS2), characterized by a very flat profile between $c a .500$ and $620 \mathrm{~nm}$. This shape becomes rounder in the EADS2 $\rightarrow$ EADS3 transition (3.4 ps), and a clearer shallow maximum builds up around $600 \mathrm{~nm}$, as expected for a $\mathrm{WH}^{\bullet+}$ radical. ${ }^{78}$ As mentioned in the case of $X / 64,{ }^{31}$ and following a hypothesis proposed by Immeln et al. for a plant cryptochrome, ${ }^{41}$ this evolution might be attributed to the cooling of $\mathrm{WH}^{\bullet+}$ in a few ps, assuming this radical is initially produced in a hot state by the very fast primary photoreduction 
of $F A D_{o x}$. Alternatively, one might attribute the unique shape of EADS2 to the proximal tryptophanyl radical $\left(\mathrm{W}_{399} \mathrm{H}^{\bullet+}\right)$, which would supposedly have a distinct absorption spectrum because of its specific environment, i.e. deeply buried inside the protein and in close Coulombic interaction with the anionic semiquinone radical $\left(F A D^{\bullet-}\right)$. According to this hypothesis, the change of shape between EADS2 and EADS3 would have to be assigned to a migration of the oxidation hole $\left(\mathrm{WH}^{\bullet+}\right)$ along the ET chain. The transitions EADS3 $\rightarrow$ EADS4 (41 ps) and EADS4 $\rightarrow$ EADS5 (630 ps) represent only very weak absorption changes in the Y373F mutant.

In the case of WT, the main difference with respect to the Y373F mutant is the final step (710 ps), which leads to a strongly decreased absorption in the red part of the spectrum (see normalized EADS5 in Figure 3). We have assigned this step to proton-coupled ET from $\mathrm{Y}_{373}$ to the terminal tryptophanyl cation radical $W_{322} \mathrm{H}^{\bullet+}$, yielding a long-lived $F A D^{\bullet-} / Y^{\bullet}$ radical pair (see Section 4.2 ). We recall that spectral changes not seen in Y373F already arise during the 55-ps phase of WT (see Figure 3 and Figure S9). This observation will be discussed below.

In order to establish more firmly the nature of EADS2 and EADS3, we fitted those spectra with a linear combination of reference spectra of $\mathrm{FAD}^{\bullet-}$ and $\mathrm{WH}^{\bullet+}$, suitably scaled and shifted (see Section 2.2.2 and Equation $\mathrm{S} 4$ in $\mathrm{SI}$, Section S5.2), and complemented by the negative $\mathrm{FAD}_{\text {ox }}$ bleaching contribution. The modification imposed on the FAD ${ }^{\bullet-}$ reference spectrum (Figure S13, SI, Section S5.2) is relatively small, while the $\mathrm{WH}^{\bullet+}$ spectrum needs to be markedly shifted to the red $(\sim 20 \mathrm{~nm})$. Such a shift, likely due to the local environment of the tryptophanyl radical inside the protein, has been reported before also for other PCSf proteins. ${ }^{31,40}$ The fits shown in Figure S12 are not perfect because the simple alterations imposed on the reference spectra cannot completely reproduce the actual FAD ${ }^{\bullet-}$ and $\mathrm{WH}^{\bullet+}$ spectra inside CraCRY. They nevertheless sufficiently support the hypothesis that EADS2 and EADS3 result from the photoinduced reduction of $F A D_{\text {ox }}$ by a tryptophan.

One may finally note for both WT and Y373F that the blue absorption and bleaching bands of EADS2 and EADS3 can be nearly superimposed (Figure 2). This suggests that almost no charge recombination of the $\mathrm{FAD}^{\bullet-} / \mathrm{WH}^{\bullet+}$ pair, expected to induce a loss of transient absorption intensity, takes place during the second kinetic phase. The main variation occurs in the red part of the spectrum, which was above suggested to derive either from a cooling of the initial $\mathrm{WH}^{\bullet+}$ radical or from the migration of the oxidation hole to a different tryptophan.

As previously mentioned, the spectral evolution from EADS3 to EADS4 in WT is nearly absent in Y373F (see Figure 3). Since a significant decay of the relative amplitude is observed around $600 \mathrm{~nm}$, the hypothesis of tyrosine oxidation during the 55 -ps step has been considered but finally set aside for lack 

the building of a $\mathrm{W}_{322} \mathrm{H}^{\bullet+}$ population characterized by a specific (weaker) absorption in the presence of $\mathrm{Y}_{373}$ (see SI, Section S5.4). As mentioned above, the 55-ps step is accompanied by partial charge recombination. According to the analysis in SI, Section 5.4, the corresponding yield is $23 \%$ (for this step only). However, given that the population losses during the preceding steps are probably small (extremely fast step one and almost no evolution of the bleaching band during step two), this number is likely close to the net yield of charge recombination counted from the initially excited flavin.

\subsubsection{Migration of the oxidation hole along the Trp triad}

In this section, we intend to use the anisotropy data to assign the $\mathrm{WH}^{\bullet+}$ spectroscopic signatures detected in the transient absorption spectra to specific residues. Following an approach previously described in Refs. ${ }^{31,40}$, we chose to probe $\mathrm{WH}^{\bullet+}$ around $610 \mathrm{~nm}$, expecting under these conditions that essentially a single transition of $\mathrm{WH}^{\bullet+}$ is observed. Table 2 gathers the corresponding values of the EAAS (noted $r_{\mathrm{i}}$ ), after initial decay of the excited state (averaged over $5 \mathrm{~nm}$ ). Within experimental errors (of the order of \pm 0.01 ), the $r_{i}^{\prime}$ 's of WT and Y373F are rather similar, with a slightly larger difference for $r_{4}$.

At this point, it is interesting to compare the experimental anisotropies to predictions based on the crystal structure of WT-CraCRY (PDB entry $5 Z M 0)^{70}$ and on known directions of the transition dipole moments of $\mathrm{FAD}_{\mathrm{ox}}$ and $\mathrm{WH}^{\bullet+}$, as previously described. ${ }^{31,40}$ Table 3 (line raw) gathers the anisotropies expected for each $\mathrm{WH}^{\bullet+}$ radical of the ET chain if one could probe them independently.

These predictions may however not be fully comparable to the experimental anisotropies because a small contribution of $\mathrm{FAD}^{\bullet-}$ could be present at $610 \mathrm{~nm}$, as suggested by the fact that EADS5 keeps a positive value in this region in spite of the decay of $\mathrm{WH}^{\bullet+}$. Such a red tail of the $\mathrm{FAD}^{\bullet-}$ spectrum has been reported before. ${ }^{79,80}$ From EAAS5 of WT in $\mathrm{D}_{2} \mathrm{O}$ (less noisy than in $\mathrm{H}_{2} \mathrm{O}$ ), and neglecting the small amount of $\mathrm{W}_{322} \mathrm{H}^{\bullet+}$ reduced in $5 \mathrm{~ns}$, the anisotropy of $\mathrm{FAD}^{\bullet-}$ at $610 \mathrm{~nm}$ is estimated to be of the order of -0.1 . If both $\mathrm{WH}^{\bullet+}$ and $\mathrm{FAD}^{\bullet-}$ contribute to the experimental anisotropy, the latter should be decomposed as follows: $:^{71}$

$$
r_{\mathrm{exp}}=x r_{\mathrm{W}}+(1-x) r_{\mathrm{F}}
$$

where $r_{\mathrm{W}}$ and $r_{\mathrm{F}}$ are the pure anisotropies respectively attached to $\mathrm{WH}^{\bullet+}$ and $\mathrm{FAD}^{\bullet-}$ and $x$ is the relative contribution of $\mathrm{WH}^{\bullet+}$ to the total isotropic signal. Taking $x=0.8$ as a crude lower limit (a value of around 0.83 may be obtained from the modified $\mathrm{WH}^{\bullet+}$ and $\mathrm{FAD}^{\bullet-}$ of Figure S9), one gets new values listed in Table 3 (line correction), which provide a qualitative idea of the effect of the FAD ${ }^{\bullet-}$ contribution on the predictions. 
It first appears that $r_{2}$ is relatively close (within errors) to the predicted anisotropy of $\mathrm{W}_{399} \mathrm{H}^{\bullet+}$ that is expected to be produced after primary reduction of the flavin by the proximal $W_{399}$ residue. The possible involvement of $\mathrm{W}_{401}$ in the first kinetic step is very unlikely since it is placed at a much larger distance of FAD (7.9 $\AA$; Figure $1 A)$ than $W_{399}(4.0 \AA)$. In EcCPDI where the equivalent tryptophan $\left(W_{384}\right)$ is only at $6.3 \AA$ from FAD, Liu et al. found that the ET from $W_{384}$ to $F A D_{o x}{ }^{*}$ takes place at a rate of $(80 \mathrm{ps})^{-1}$ in a mutant lacking the proximal tryptophan. ${ }^{37}$ An even slower rate is to be expected in CraCRY.

During the next step, the anisotropy changes sign and $r_{3}$ becomes positive as expected for $\mathrm{W}_{376} \mathrm{H}^{\bullet+}$, the medial aromatic residue of the canonical tryptophan triad, however, without reaching its predicted value. Since $W_{332}$ is situated at a very close distance from $W_{399}(3.8 \AA$; Figure $1 A)$, it is possible that $W_{332}$ effectively competes with $W_{376}$ for the reduction of $\mathrm{W}_{399} \mathrm{H}^{\bullet+}$, as previously reported for EcCPDI by Li et al. ${ }^{37}$ The resulting $\mathrm{W}_{332} \mathrm{H}^{\bullet+}$, expected to have a slightly less positive anisotropy than $\mathrm{W}_{376} \mathrm{H}^{\bullet+}($ Table 3 ), is then thought to contribute to the observed $r_{3}$. In addition, the driving force of ET from $W_{376}$ to $W_{399} \mathrm{H}^{\bullet+}$ may be small, as claimed by Li et al., ${ }^{37}$ for the equivalent residues in EcCPDI, so that $r_{3}$ might be diminished by a negative contribution from $\mathrm{W}_{399} \mathrm{H}^{\bullet+}$ still present at the end of the second ET step. Finally, one may also hypothesize that $r_{3}$ takes a negative contribution from the distal $\mathrm{W}_{322} \mathrm{H}^{\bullet+}$, meaning that delocalization of the oxidation hole has already reached the last tryptophan of the triad at this step of the reaction. In any case, it appears that the oxidation hole is never wholly localized on $W_{376}$.

The values of $r_{4}$ and $r_{5}$ are more readily understood in the case of Y373F. We see that $r_{4}$ is quite close to the expectation for $\mathrm{W}_{322} \mathrm{H}^{\bullet+}$, and that the match becomes even better for $r_{5}$. This suggests that the third kinetic step (41 ps in Y373F) results in a dominant localization of the oxidation hole on the $W_{322}$ site. The last step (630 ps) could either sign the final localization of the oxidation hole on $W_{322}$, if it were not already completed and/or a rearrangement of the protein that stabilizes the $F A D^{\bullet-} / \mathrm{W}_{322} \mathrm{H}^{\bullet+}$ pair (possibly required for a complete localization of the oxidation hole on $\mathrm{W}_{322}$ ) and eventually blocks the weak charge recombination channel still observed during this step. In the case of WT, the fourth and possibly third kinetic steps are marked by the oxidation of the $Y_{373}$ residue. Since $Y \bullet$ does not contribute at $610 \mathrm{~nm}$, Equation 3 would still apply but the $x$ ratio would be smaller than in the absence of $Y^{\bullet}$. In the limiting case of $r_{5}$, one may assume that the amount of $\mathrm{WH}^{\bullet+}$ is relatively small and take $x=0$ as a rough approximation. This leads to the estimation of $r_{\mathrm{F}}$ mentioned above $\left(-0.1\right.$; from WT in $\left.\mathrm{D}_{2} \mathrm{O}\right)$. The case of $r_{4}$ is interesting because Equation 3 would predict a value situated somewhere between ca. -0.06 and -0.1 (depending on $x$ ) provided the oxidized tryptophan is $W_{322}$ (see Table 3, line raw). The fact that $r_{4}$ has a less negative value than that (Table 2; WT) suggests that the localization of the oxidation hole on $\mathrm{W}_{322}$ is 

begins while there is still a certain degree of delocalization over different tryptophans. It might reciprocally be speculated that the replacement of tyrosine by phenylalanine somehow enhances protein and water reorganization leading to full localization of the oxidation hole on $W_{322}$ during the third kinetic step, as observed for Y373F.

\subsection{Oxidation of tyrosine $Y_{373}$}

\subsubsection{Kinetics and spectral changes}

In our attempts to establish the kinetics and the mechanism of oxidation of tyrosine $\mathrm{Y}_{373}$ in $\mathrm{CraCRY}$, we focused on kinetic and spectral features that depended on the presence of this tyrosine residue, i.e. were absent or strongly modified in the Y373F mutant.

Our results obtained by both pump-probe and real-time TAS agree that reduction of a tryptophan cation radical $\mathrm{WH}^{\bullet+}$ occurs essentially with a time constant of $~ 600-700$ ps in WT CraCRY (see Figures 3A, $5 \mathrm{~A}$ and S2 (A-C)), while the same $\mathrm{WH}^{\bullet+}$ species lives for $\sim 250$ nanoseconds in the Y373F mutant (Figures $6(C, D)$ and S3). About $20 \%$ of this reduction is due to recombination with $F A D^{\bullet-}$ (observed as a partial recovery of the $F A D_{o x}$ bleaching band of around $450 \mathrm{~nm}$; see Figures $3 \mathrm{~A}$ and $5 \mathrm{~A}$ ) and about $80 \%$ should hence be due to electron transfer from $\mathrm{Y}_{373}$. Real-time TAS detected in addition a minor slower $\mathrm{WH}^{\bullet+}$ reduction phase with a time constant of $\sim 5 \mathrm{~ns}$ and approx. one third of the amplitude of the $\sim 600-700 \mathrm{ps}$ phase. Neither variation of the $\mathrm{pH}$ (between 6.5 and 9.0; Figure S2C) nor use of a deuterated buffer solution (Figure S8) had a significant effect on the reduction kinetics of $\mathrm{WH}^{\bullet+}$.

Looking for a direct evidence of ultrafast tyrosine oxidation, we used the spectrally-resolved ultrafast TAS data and focused on the fourth time component, fitted by a lifetime of 710 ps (transition from EADS4 to EADS5). In order to characterize the spectral changes due to the reactive fraction of the transient population, i.e. excluding charge recombination, EADS5 was fitted with a weighted sum of EADS4, $\mathrm{WH}^{\bullet+}$ and $\mathrm{Y}^{\bullet}$ contributions, as detailed in SI, Section S5.3 (Equation S5). The result shown in Figure 6B (in EADS5 - $\phi$ EADS4 form, where $1-\phi$ is the yield of charge recombination occurring during the EADS4 $\rightarrow$ EADS5 step) provides a convincing support that $\mathrm{WH}^{\bullet+}$ indeed undergoes reduction by a tyrosine. The optimized parameters are available in SI, Section S5.3 (Table S2) and the modified reference spectra are presented in Figure $6 \mathrm{C}$. The $\mathrm{WH}^{\bullet+}$ and $\mathrm{Y}^{\bullet}$ spectra providing the best fit of our experimental data are found to be both red-shifted relative to solution spectra, by 25 and $8 \mathrm{~nm}$, respectively (Figure $6 \mathrm{C}$ ). It is worth noting that a 9-nm red shift of the $\mathrm{Y}^{\bullet}$ spectrum, as compared to a 
reference spectrum in water, was also reported by Oldemeyer et al. in their time-resolved spectroscopic study. ${ }^{24}$ The fit also indicates that $F A D^{\bullet-} / \mathrm{WH}^{\bullet+}$ recombination (competing with the oxidation of $\mathrm{Y}_{373}$ ) occurs with a yield of $22 \%$. We similarly attempted to fit EADS4 according to Equation S5 to check the hypothesis of early oxidation of $\mathrm{Y}_{373}$ (see Section 4.1.1). This approach however led to a conflicting result, a large blue shift of the modified $Y^{\bullet}$ spectrum, and was not considered conclusive (see more details in SI, Section S5.4).

The kinetics of tyrosine oxidation should show up in our sub-ns/ns real time TAS data at $405 \mathrm{~nm}$ (within the absorption band of $Y^{\bullet}$ ). The absorption rise due to the formation of $Y^{\bullet}$ may, however, be compensated of even overcompensated by the partial recombination of $F A D^{\bullet-} / \mathrm{WH}^{\bullet+}$ that is accompanied by an absorption decrease at $405 \mathrm{~nm}$ (see spectra in Figure 1B). Such a compensation is apparent in the ultrafast TAS data: while the uncorrected amplitudes around $400 \mathrm{~nm}$ slightly decrease from 10 ps to 3.2 ns, (Figure S4 (B,C) and Figure S7), there is an increase after correction for recombination of $\mathrm{FAD}^{\bullet-} / \mathrm{WH}^{\bullet+}$ (compare NEADS3, 4 and 5 in Figure $\left.3 \mathrm{~A}\right)$. Similarly, no rise with $\tau \approx 600$ ps was observed by real-time TAS at $405 \mathrm{~nm}$ (black trace in Figure 4A). A minor rise with $\tau \approx 5 \mathrm{~ns}$, in line with the slower phase of $\mathrm{WH}^{\bullet+}$ reduction, is however visible.

In an attempt to reconstruct the kinetics of tyrosine oxidation from the real-time TAS trace at $405 \mathrm{~nm}$ by correcting for the $F A D^{\bullet-}$ contribution, we assumed that essentially only $F A D_{o x}, F A D^{\bullet-}$ and $Y^{\bullet}$ contribute at $405 \mathrm{~nm}$ (neglecting the weak contribution from $\mathrm{WH}^{\bullet+}$; see Figure $1 \mathrm{~B}$ ) and that the kinetics of $\mathrm{FAD}^{\bullet-}$ population is represented by the kinetics of the bleaching at $457 \mathrm{~nm}$ (blue trace in Figure $4 \mathrm{~A}$; here we also neglect a weak contribution from $\mathrm{WH}^{\bullet+}$ ). Subtraction of the $457 \mathrm{~nm}$ trace (inverted and rescaled to the same initial amplitude) from the $405 \mathrm{~nm}$ trace yielded a "corrected" $405 \mathrm{~nm}$ signal (Figure 6A) that we consider to be a coarse approximation of the kinetics of tyrosine oxidation (see SI, Section S2.1 for more details on how the corrected $405 \mathrm{~nm}$ signal was constructed). A biexponential fit of the corrected signal (using Equation S2) yielded time constants of 820 ps (with an amplitude of 6 $\mathrm{mOD}$ ) and $6.0 \mathrm{~ns}$ (with an amplitude of $\sim 3 \mathrm{mOD}$ ). The tyrosine oxidation hence follows essentially the same biphasic kinetics as $\mathrm{WH}^{\bullet+}$ reduction ( $\sim 600-700 \mathrm{ps}$ and $\sim 5 \mathrm{~ns}$ ) observed above $500 \mathrm{~nm}$, confirming that $\mathrm{Y}_{373}$ is oxidized by a tryptophanyl cation radical, most likely the distal $\mathrm{W}_{322} \mathrm{H}^{\bullet+}$.

Let us finally emphasize that, according to our spectral analysis (Figure 6B), the oxidized tyrosine is observed in its deprotonated $\mathrm{Y}^{\bullet}$ form. The protonated $\mathrm{YH}^{\bullet+}$ radical cation has recently been reported in a flavoenzyme to have a strongly displaced spectrum around $490 \mathrm{~nm}^{81}$, which would not match our observations (see also the lack of induced absorption at $488 \mathrm{~nm}$ in the real-time TAS data; green trace in Figure 4A). Tyrosyl deprotonation is thus intimately involved in the process that must be considered a 

by PCET from $\mathrm{Y}_{373}$ and approximately four times slower recombination with $\mathrm{FAD}^{\bullet-}$. The intrinsic rate constant of the major phase of PCET would thus be of the order of $(800-900 \text { ps })^{-1}$. We will adopt an approximate value of $(800 \mathrm{ps})^{-1}$ for the discussion of the PCET mechanism presented in the following section.

\subsubsection{Reaction mechanism of tyrosine oxidation}

As discussed above, the reduction of $\mathrm{WH}^{\bullet+}$ by PCET from $\mathrm{Y}_{373}$ in CraCRY mostly proceeds with an intrinsic rate constant of $(800 \mathrm{ps})^{-1}$. This is substantially faster than the fastest phase of oxidation of the so-called tyrosine-Z in oxygen-evolving PSII ( $\tau \sim 30 \mathrm{~ns}$ ). Remarkably, in both cases the reaction kinetics was found to be independent of pH (between 6.5 and 9.0 in CraCRY (Figure S2C) and between 4.5 and 7.5 in PSII82) and was not significantly affected by deuteration of the buffer solution (Table 1). ${ }^{83-85}$

The structure of CraCRY (Figure 1A) strongly suggests that the electron acceptor is $\mathrm{W}_{322} \mathrm{H}^{\bullet+}$, the distal member of the canonical Trp triad, because it is located at only $3.8 \AA$ edge-to-edge distance from $Y_{373}$. The proton acceptor is likely to be aspartate $D_{321}$ that forms a hydrogen bond with the $Y_{373}$ hydroxyl group (3.0 А; Figure 1A). ${ }^{70}$

In the following, we discuss possible reaction mechanisms in the framework of limiting cases of PCET following the classification by Hammes-Schiffer and Stuchebrukhov ${ }^{64}$ and Liu et al. ${ }^{86}$ The reaction pathways considered for CraCRY and our notations are shown in Scheme 1. We note that based on published reduction potentials and $\mathrm{p} K_{\mathrm{a}}$ values in aqueous solution of the amino acid side chains involved, namely $E^{\circ}\left(\mathrm{WH}^{\bullet+} / \mathrm{WH}\right) \approx 1.07 \mathrm{~V}$ vs. $\mathrm{NHE}^{54} \quad E^{\circ}\left(\mathrm{YH}^{\bullet+} / \mathrm{YH}\right) \approx 1.35 \mathrm{~V}^{54} \quad E^{\circ}\left(\mathrm{Y}^{\bullet} / \mathrm{Y}^{-}\right) \approx 0.65 \quad \mathrm{~V}^{54}$ $\mathrm{p} K_{\mathrm{a}}\left(\mathrm{YH}^{\bullet+} / \mathrm{Y}^{\bullet}\right) \approx-2,{ }^{60} \mathrm{p} K_{\mathrm{a}}\left(\mathrm{YH} / \mathrm{Y}^{-}\right) \approx 9.9,^{54}$ and $\mathrm{p} K_{\mathrm{a}}\left(\mathrm{DH} / \mathrm{D}^{-}\right)=3.9^{87}$, the overall PCET reaction would be downhill by $0.07 \mathrm{eV}$. ET as the first step would, however, be uphill by $0.28 \mathrm{eV}$ and PT as the first step even by $0.35 \mathrm{eV}$. As discussed below, these values may be modified considerably by the protein environment and by interactions between the species.

(I) Sequential ET/PT (blue pathway in Scheme 1)

(la) $\quad \mathrm{PT}$ is "fast" $\left(k_{\mathrm{PT}}>>k_{\mathrm{BET}}\right)$

PT takes immediately place when the "activated" state $\left[\mathrm{W}_{322} \mathrm{H} \mathrm{Y}_{373} \mathrm{H}^{\bullet+\ldots} \mathrm{D}_{321}{ }^{-}\right]$is reached by uphill ET. The uphill ET is hence rate limiting.

$$
k_{\mathrm{PCET}}=k_{\mathrm{FET}}
$$

(Ib) $\quad \mathrm{PT}$ is "slow" $\left(k_{\mathrm{PT}}<<k_{\mathrm{BET}}\right)$ 
Several ET "attempts" are needed to complete the reaction by PT. In other words, PT occurs from an ET pre-equilibrium in which the "activated" state $\left[\mathrm{W}_{322} \mathrm{H} \mathrm{Y}_{373} \mathrm{H}^{\bullet+\ldots} \mathrm{D}_{321}{ }^{-}\right]$has low population.

$$
k_{\mathrm{PCET}}=k_{\mathrm{PT}} k_{\mathrm{ET}} \text { with } k_{\mathrm{ET}}=k_{\mathrm{FET}} / k_{\mathrm{BET}}=\exp \left(-\Delta G_{\mathrm{ET}}^{\circ} / k_{\mathrm{B}} T\right)
$$

(II) Sequential PT/ET (red pathway in Scheme 1)

(Ila) ET is "fast" $\left(k_{\mathrm{ET}}>>k_{\mathrm{BPT}}\right)$

The ET takes place immediately when the "activated" state $\left[\mathrm{W}_{322} \mathrm{H}^{\bullet+} \mathrm{Y}_{373}-\cdots \mathrm{HD}_{321}\right]$ is reached by the uphill PT. The uphill PT is hence rate limiting.

$k_{\mathrm{PCET}}=k_{\mathrm{FPT}}$

(IIb) $\quad \mathrm{ET}$ is "slow" $\left(k_{\mathrm{ET}}<<k_{\mathrm{BPT}}\right)$

Several PT "attempts" are needed to complete the reaction by the ET. In other words, the ET occurs from a PT pre-equilibrium in which the "activated" state $\left[\mathrm{W}_{322} \mathrm{H}^{\bullet+} \mathrm{Y}_{373}-\ldots \mathrm{HD}_{321}\right]$ has low population.

$$
k_{\mathrm{PCET}}=k_{\mathrm{ET}} K_{\mathrm{PT}} \text { with } K_{\mathrm{PT}}=k_{\mathrm{FPT}} / k_{\mathrm{BPT}}=\exp \left(-\Delta G_{\mathrm{PT}}^{\circ} / k_{\mathrm{B}} T\right)
$$

(III) Concerted PCET (green pathway in Scheme 1)

ET and PT occur together in one step, avoiding formation of a high energy intermediate. No simple rate equation can be given.

Among these cases, Ib and Ila are expected to show a significant normal H/D KIE because the rate of forward PT enters directly into the rate equation. For concerted PCET (case III), a substantial normal KIE is considered to be a hallmark. ${ }^{6+^{+\dagger}}$ Because of the absence of a significant KIE for $\mathrm{W}_{322} \mathrm{H}^{\bullet+}$ reduction by $\mathrm{Y}_{373}$ in CraCRY, we focus on the two remaining cases.

For sequential ET/PT with "fast" PT (case la), the overall rate is given by the (uphill) forward ET. The expected rate of this ET step at room temperature may be estimated using the following semi-empirical expression for the rate $k_{\mathrm{ET}}^{e n}\left(\right.$ in s $^{-1}$ ) of endergonic ET in proteins at room temperature: ${ }^{89}$

$$
\log k_{\mathrm{ET}}^{e n}=13.0-(1.2-0.8 \rho)(R-3.6)-3.1\left(-\Delta G^{\circ}+\lambda\right)^{2} / \lambda-\Delta G^{\circ} / 0.06
$$

where $R$ is the edge-to-edge distance between electron donor and acceptor (in $\AA$ ), $\Delta G^{\circ}$ the (positive) standard Gibbs free energy (in eV) of the ET and $\lambda$ the reorganization energy (in eV). $\rho$ represents the packing density of protein atoms in the volume between electron donor and acceptor.

\footnotetext{
It A recent theoretical work ${ }^{88}$ presented special cases with negligible KIE, in which highly excited (several eV) electron-proton vibronic states dictated the dynamics. Such highly excited states are not expected to play a role in PCET between tyrosine and tryptophan in CraCRY.
} 
Using $R=3.8 \AA$ (Figure 1A), $\Delta G^{\circ}=0.28 \mathrm{eV}$ (see above), $\lambda=1 \mathrm{eV}$ (considered as "unremarkable" in Ref. ${ }^{89}$ and $\rho=0.76$, an average value for ET reactions in proteins, ${ }^{89}$ Equation 4 yields $k_{\mathrm{ET}}^{e n}=(250 \mathrm{~ns})^{-1}$, i.e. more than two orders of magnitude slower than the rate of $\sim(800 \mathrm{ps})^{-1}$ as deduced from the observed kinetics (see above). The somewhat arbitrarily chosen value of $\rho$ is not critical in this evaluation because the term $(R-3.6)$ is small. The also not well known $\lambda$ has more impact, but even when choosing the optimal value $\lambda=\Delta G^{\circ}$, the calculated rate of $(6.1 \mathrm{~ns})^{-1}$ is still slower by an order of magnitude than the observed rate. Most critical in this evaluation is the endergonic degree of the reaction. Keeping $\lambda=1 \mathrm{eV}$, a value of $\Delta G^{\circ}=0.0642 \mathrm{eV}$ would be required to get a rate of $(800 \mathrm{ps})^{-1}$, i.e. far below the value of 0.28 $\mathrm{eV}$ estimated above. We however do not exclude that the protein environment and the interactions between the species may have such a strong effect on the energetics, for the following reasons:

(1) The reduction potential of $\mathrm{WH}^{\bullet+} / \mathrm{WH}$ embedded in the protein matrix is expected to be higher than in aqueous solution (1.07 V) because the positive charge is less stabilized in a low dielectric environment. E.g., the reduction potentials of members of the canonical Trp triad in oxidized EcCPDI were estimated to $\geq 1.48,1.41$ and $1.24 \mathrm{~V}$ vs $\mathrm{NHE}^{37}$ in the order of increasing distance from $F A D$, similar to estimations that can be deduced from the energetics in semi-reduced EcCPDI. ${ }^{36}$ The large drop from the medial to the distal Trp in EcCPDI can be understood as a stabilization of the positive charge by the proximity of the aqueous phase ${ }^{36,37}$ and may be less pronounced in CraCRY because of the presence of $\mathrm{Y}_{373}{ }^{\neq \neq}$

(2) The negative charge of aspartate $D_{321}{ }^{-}$in close proximity of $Y_{373}$ may decrease the $\mathrm{YH}^{\bullet+} / \mathrm{YH}$ reduction potential due to electrostatic stabilization of the positive charge.

Case la further requires that the subsequent PT is much faster than back $\mathrm{ET}\left(k_{\mathrm{PT}}>>k_{\mathrm{BET}}\right)$. Back ET would be exergonic and its rate may be estimated by Equation 5.89

$$
\log k_{\mathrm{ET}}^{e x}=13.0-(1.2-0.8 \rho)(R-3.6)-3.1\left(\Delta G^{\circ}+\lambda\right)^{2} / \lambda
$$

Using $\Delta G^{\circ}=-0.0642 \mathrm{eV}, \lambda=1 \mathrm{eV}$ and the other parameters as above, one obtains $k_{\mathrm{BET}}=k_{\mathrm{ET}}^{e x}=(68 \mathrm{ps})^{-}$ 1.

Based on the solution $\mathrm{p} K_{\mathrm{a}}$ values given above, PT from $\mathrm{Y}_{373} \mathrm{H}^{\bullet+}$ to $\mathrm{D}_{321}{ }^{-}$would be downhill by $0.35 \mathrm{eV}$. The PT distance would be at least $1.0 \AA \AA$ based on the $3.0 \AA \AA$ hydrogen bond length in the crystal structure (Figure $1 \mathrm{~A}$ ) and assuming an $\mathrm{O}-\mathrm{H}$ distance of $1.0 \AA$ both in the reactant $\mathrm{Y}_{373} \mathrm{H}^{\bullet+}$ and in the product $\mathrm{D}_{321} \mathrm{H}$. Proton transfer in hydrogen bonds can be as fast as tens of femtoseconds over a distance of approx.

\footnotetext{
$\ddagger \ddagger$ Our data by ultra-fast spectroscopy indicate that $\mathrm{W}_{322} \mathrm{H}^{\bullet+}$ is less stabilized in the WT than in the Y373F mutant; see Section 4.1.2.
} 
$0.6 \AA$ in the excited state of 1-hydroxy-2-acetonaphtone ${ }^{90}$ or at most a few picoseconds over approx. $0.7 \AA\left(\mathrm{O}-\mathrm{H} \cdots \mathrm{O}\right.$ hydrogen bond length of $2.7 \AA$ ) in green fluorescent protein ${ }^{91,92}$ but depends very strongly on the transfer distance, decreasing typically by one order of magnitude per $0.07 \AA .93$ It is hence questionable that proton transfer from $\mathrm{Y}_{373} \mathrm{H}^{\bullet+}$ to $\mathrm{D}_{321}{ }^{-}$can be faster than back ET from $W_{322}$ to $\mathrm{Y}_{373} \mathrm{H}^{\bullet+}$. We note, however, that formation of $\mathrm{Y}_{373} \mathrm{H}^{\bullet+}$ may induce structural changes (e.g. due to electrostatic interaction with $\mathrm{D}_{321}{ }^{-}$) that decrease the proton transfer distance relative to the (dark state) crystal structure.

For sequential PT/ET with "slow" ET (case Ilb), the overall rate is determined by the product of the PT (pre-)equilibrium constant $K_{\mathrm{PT}}$ and the rate constant $k_{\mathrm{ET}}$ of the subsequent $\mathrm{ET}$. There should be no significant KIE, ${ }^{64}$ because the effects on the forward and backward PT are usually similar and cancel each other out in the rate expression. ${ }^{\S \S}$

Based on the solution values given above, PT from $\mathrm{Y}_{373} \mathrm{H}$ to $\mathrm{D}_{321}{ }^{-}$would be uphill by $\Delta G_{\mathrm{PT}(1)}^{\circ}=0.35 \mathrm{eV}$ and $K_{\mathrm{PT}}=10^{-6}$. Subsequent ET from $\mathrm{Y}_{373^{-}}$to $\mathrm{W}_{322} \mathrm{H}^{\bullet+}$ would be downhill by $0.42 \mathrm{eV}$, yielding an ET rate of (1.5 ps) $)^{-1}$ according to Equation 5 with the other parameters as above, and an overall rate of $(1.5 \mu \mathrm{s})^{-1}$. Assuming the optimal reorganization energy $\lambda=-\Delta G^{\circ}=0.42 \mathrm{eV}$, the ET rate would be $(130 \mathrm{fs})^{-1}$ and the overall rate $(130 \mathrm{~ns})^{-1}$, still very far off the experimental rate of $(800 \mathrm{ps})^{-1}$. To obtain the rate of (800 ps) ${ }^{-1}$, the PT (pre-)equilibrium constant would have to be $>1.6 \times 10^{-4}$, corresponding to $\Delta G_{\mathrm{PT}}^{\circ}<0.22$ $\mathrm{eV}$ and a $\mathrm{p} K_{\mathrm{a}}$ difference $<3.8$ (as compared to 6.0 according to solution values; see above). We would not exclude such a strong modification of the PT equilibrium, because:

(1) $p K_{a}$ values of amino acid side chains in proteins can deviate substantially from solution values. ${ }^{87}$

(2) The presence of $W_{322} \mathrm{H}^{\bullet+}$ close to $Y_{373}$ is likely to stabilize $\mathrm{Y}_{373}{ }^{-}$by electrostatic interaction and hence to decrease the endergonicity of PT from $Y_{373}$ to $D_{321}{ }^{-}$.

Case IIb further requires that (downhill) back PT is much faster than $\mathrm{ET}\left(k_{\mathrm{ET}}<<k_{\mathrm{BPT}}\right)$, i.e. well in the subpicosecond range. As discussed above for case la, it is questionable that such a fast proton transfer is possible at the distance in the (dark-state) crystal structure. One should, however, not exclude the possibility that formation of $W_{322} \mathrm{H}^{\bullet+}$ induces a shortening of the hydrogen bond between $\mathrm{Y}_{373}$ and $D_{321}$.

Our experimental data indicated that a minor fraction of $Y_{373}$ was oxidized at a slower rate of $\sim(5 \mathrm{~ns})^{-1}$. This may reflect some conformational heterogeneity of CraCRY that affects ET or PT distances or energetics, but may also be explained by an energetic relaxation of $\mathrm{W}_{322} \mathrm{H}^{\bullet+}$ due to reorganization of

\footnotetext{
$\S$ A remarkable exception was recently reported for PCET involving a metal hydride with an appended pyridine base: a substantial inverse KIE was observed and attributed to an isotope effect on the PT (pre)equilibrium constant due to differences in the zero point energy of the metal- $\mathrm{H}$ and $\mathrm{H}^{+}$-pyridine vibrations. ${ }^{86}$
} 


\footnotetext{
${ }^{* * *}$ Concerted PCET has been suggested for tyrosine oxidation in PSII with inactivated oxygen evolution, ${ }^{49}$ where the environment of tyrosine- $\mathrm{Z}$ is substantially modified. ${ }^{51,53}$ In this case tyrosine oxidation by $\mathrm{P} 680^{\bullet+}$ is slower and $\mathrm{pH}$ dependent (time constants from $\sim 300 \mathrm{~ns}$ at $\mathrm{pH} 9$ to $\sim 50 \mu \mathrm{s}$ at $\mathrm{pH} 4^{95,96}$ and shows a significant normal H/D KIE at $\mathrm{pH}<8 .{ }^{97-99}$
}

arrow in Scheme 1). Relaxed $\mathrm{W}_{322} \mathrm{H}^{\bullet+}$ would have a lower reduction potential, and hence, for sequential ET/PT (case la), ET would be more uphill and the overall reaction would slow down. In the sequential PT/ET scenario (case IIb), ET would be less downhill and the overall reaction would slow down as well (except if ET occurred in the Marcus inverted region (i.e. $-\Delta G^{\circ}>\lambda$ ), which is highly unlikely given the moderate driving force). The free energy decrease due to relaxation would have to be in the order of 0.07 and $0.2 \mathrm{eV}$ in cases la and IIb, respectively, to account for a slowdown of the overall reaction to $\sim 5$ ns.

We finally would like to compare tyrosine oxidation in CraCRY with oxidation of tyrosine-Z by P680*+ in oxygen evolving PSII that has been studied experimentally for 40 years and is still the subject of controversial discussions. ${ }^{48-53}$ The fastest kinetics in PSII has a time constant of $\sim 30 \mathrm{~ns}^{56-58}$ and does not show a significant KIE. ${ }^{83-85}$ The reduction potential of $\mathrm{P} 680^{\circ+} / \mathrm{P} 680$ is $\sim 1.25 \mathrm{~V},{ }^{55}$ i.e. $0.1 \mathrm{~V}$ below the solution potential of $\mathrm{YH}^{\bullet+} / \mathrm{YH}$, and probably even more below that of $\mathrm{Y}_{2} \mathrm{H}^{\bullet+} / \mathrm{Y}_{2} \mathrm{H}$ in a protein. ${ }^{54}$ The edgeto-edge distance between $\mathrm{P} 680$ and $\mathrm{Y}_{\mathrm{Z}}$ is $9.0 \AA \AA^{, 53}$ as compared to $3.8 \AA$ between $\mathrm{Y}_{373}$ and $\mathrm{D}_{321}$ in $\mathrm{CraCRY}$. $\mathrm{Y}_{\mathrm{Z}}$ forms a strong $\mathrm{H}$-bond with a histidine residue (O-N distance, $2.5 \AA,^{94}$ as compared to $3.0 \AA$ for the hydrogen bond between $\mathrm{Y}_{373}$ and $\mathrm{D}_{321}$ in $\mathrm{CraCRY}$ ). As for tyrosine oxidation in CraCRY, the absence of a significant KIE suggests either a sequential ET/PT with PT being "fast" (case la) or a sequential PT/ET with ET being "slow" (case IIb) ${ }^{* * *}$. Case la seems, however, to be excluded in PSII because the rate limiting uphill ET from $\mathrm{Y}_{\mathrm{Z}}$ to $\mathrm{P} 680^{\circ+}$ is expected to take $\geq 500$ ns according to Equation 4 using $\rho=0.75, R=9 \AA, \lambda$ $=0.77 \mathrm{eV}$ (all from Ref. ${ }^{53}$ ) and $\Delta G^{\circ} \geq 0.1 \mathrm{eV}$. A recent analysis suggested sequential PT/ET with a PT (preequilibrium constant of 0.16 and an ET rate of $(7.7 \mathrm{~ns})^{-1} .{ }^{53} \mathrm{QM} / \mathrm{MM}$ calculations on PSII indicate that the two energy minima of the proton location are very shallow and only $\sim 0.4 \AA$ apart, ${ }^{100}$ presumably allowing ultrafast and nearly activationless PT.

For the even faster tyrosine oxidation in CraCRY, the substantially longer hydrogen bond between $\mathrm{Y}_{373}$ and $\mathrm{D}_{321}$ in the dark state crystal structure does not support a sequential PT/ET mechanism unless formation of $\mathrm{W}_{322} \mathrm{H}^{\bullet+}$ substantially shortens this bond and shifts the PT (pre-)equilibrium towards $\mathrm{Y}_{373^{-}}$ ...HD $\mathrm{H}_{321}$ (see above). The very short ET distance in CraCRY, however, could enable sequential ET/PT, 
provided that the protein environment decreases the endergonic character of ET from $\mathrm{Y}_{373} \mathrm{H}$ to $\mathrm{W}_{322} \mathrm{H}^{\bullet+}$ relative to the solution value (see above).

Tyrosine oxidation during photoactivation of the SIr 1694 BLUF domain of Synechocystis PCC6803 occurs by sequential ET (from $\mathrm{Y}_{8}$ to the photoexcited FAD cofactor; edge-to-edge distance, $3.5 \AA$ ) and PT (from $\mathrm{Y}_{8} \mathrm{H}^{\bullet+}$ to $\mathrm{FAD}^{\bullet-}$ via $\mathrm{Q}_{50}$ ) on a time scale of tens of picoseconds, ${ }^{61}$ i.e. two orders of magnitude faster than tyrosine oxidation by $\mathrm{W}_{322} \mathrm{H}^{\bullet+}$ in CraCRY. A main cause of the much faster kinetics is likely the much more favorable energetics of ET in SIr 1694 BLUF, which was estimated to be downhill because of the high energy of $\mathrm{FAD}_{\mathrm{ox}}{ }^{*},{ }^{61,101,102}$ but uphill in the oxidation of $\mathrm{Y}_{373}$ in CraCRY. The term PCET has been used for the overall process of tyrosine oxidation during photoactivation of SIr1694 BLUF, ${ }^{63,67}$ despite ET as the first step of the sequential ET/PT being downhill (cf. Introduction). A very recent work named it electron-coupled double proton transfer. ${ }^{102}$

\subsection{Fate of the $F A D^{\bullet-} / Y_{373^{\bullet}}$ pair and efficiency of $F A D$ photoreduction}

The light-induced ET and PT reactions leading to FAD reduction occur in competition with nonproductive recombination (see Scheme 1). The quantum yield of FAD reduction can be estimated from the time course of the amplitude of the bleaching of the main absorption band of oxidized FAD around $450 \mathrm{~nm}$. Our data by ultrafast TAS indicate that in WT CraCRY $40-45 \%$ of the initially formed FAD ${ }^{\bullet-}$ (represented by EADS2 in Fig. $3 A$ ) is lost during formation of the $F A D^{\bullet-} / Y_{373}$ pair (represented by EADS5 in Fig. 3A). No significant further losses were detected by real time TAS up to $70 \mu$ s (Fig. 6). The quantum yield of the initial ET from $W_{399}$ to excited FAD $\left({ }^{1} \mathrm{FAD}_{\text {ox }}{ }^{*}\right)$ in $\sim 0.4$ ps is likely to be virtually $100 \%$ as this process is several orders of magnitude faster than the lifetime of singlet excited flavins in the absence of quenching by ET (a few $\mathrm{ns}^{103}$ ). We conclude that the $\mathrm{FAD}^{\bullet-} / \mathrm{Y}_{373^{\bullet}}$ pair is formed with a quantum yield of $\sim 60 \%$.

The $\mathrm{FAD}^{\bullet-} / \mathrm{Y}_{373^{\bullet}}$ pair in isolated WT CraCRY at $\mathrm{pH} 8.4$ finally decayed by recombination in tens of milliseconds (Figure 5B, inset), in line with a previous report. ${ }^{29}$ We found that this recombination is in competition with protonation of $\mathrm{FAD}^{\bullet-}$ to form the neutral, red light-absorbing radical $\mathrm{FADH} \mathrm{H}^{\bullet}$ that is the primary target for CraCRY-based red light responses of Chlamydomonas cells. ${ }^{21,22,104}$ The protonation was accelerated and hence more efficient at lower pH (see Figure S2D and SI, Section S2.2 for more details), explaining a previous observation ${ }^{23}$ that the amplitude of blue light-induced very long-lived bleaching of the FAD absorption band in the blue (attributed to formation of $F A D H^{\bullet}$ ) increased strongly with decreasing $\mathrm{pH}$. One would expect that the $\mathrm{FADH} \bullet / \mathrm{Y}_{373} \bullet$ pair recombines via activated re-formation 


\section{CONCLUDING REMARKS}

The main result of the present study is our demonstration of a very fast photoinduced proton-coupled electron transfer (PCET) ${ }^{64}$ from tyrosine to tryptophan in WT-CraCRY, forming the neutral (deprotonated) tyrosyl radical of $\mathrm{Y}_{373^{\circ}}$ in $\sim 800$ ps without significant H/D KIE. This PCET step is about 40 times faster than formation of the tyrosyl radical $Y_{Z} \bullet$ in oxygen evolving PSII. Our results may either be explained by a sequential ET/PT with fast PT or a sequential PT/ET with slow ET (see detailed discussion in Section 4.2.2). Quantum mechanical/molecular mechanical ( $Q M / M M)$ calculations may provide crucial information to decide which mechanism is actually preferred by CraCRY and how it is implemented. In particular, it would be useful to estimate the rate of PT from $\mathrm{Y}_{373} \mathrm{H}^{\bullet+}$ to $\mathrm{D}_{321}{ }^{-}$in order to evaluate whether sequential ET/PT as fast as $800 \mathrm{ps}$ and not rate-limited by PT is possible. As far as the validity of PT/ET is concerned, it would be interesting to determine if the formation of $\mathrm{W}_{322} \mathrm{H}^{\bullet+}$ could induce any shortening of the hydrogen bond between $Y_{373}$ and $D_{321}$, thereby allowing a very fast back PT from $\mathrm{D}_{321} \mathrm{H}$ to $\mathrm{Y}_{373}{ }^{-}\left(k_{\mathrm{ET}}<<k_{\mathrm{BPT}}\right.$; see Section 4.2.2), which in turn could ensure the lack of a KIE. Further experimental developments should involve checking the identity of the proton acceptor by point mutation of $D_{321}$ to a non-H-acceptor residue and studies of the temperature dependence of the tyrosine oxidation kinetics for estimating the barrier of the putative uphill step in sequential PCET. 
It is finally worth recalling that CraCRY is uniquely characterized by its ability to function as a red light receptor in vivo and to accumulate $\mathrm{FADH}^{\bullet}$ in vitro even in the (alleged) absence of external reductant. ${ }^{20}$ Our results demonstrate that the $\mathrm{FAD}^{\bullet-} \mathrm{Y}_{373} \bullet$ pair is formed with a high quantum yield $(\sim 60 \%)$ and has a long intrinsic lifetime ( $\sim 50 \mathrm{~ms}$ due to recombination). The semi-reduced FAD may therefore be easily stabilized by protonation of $\mathrm{FAD}^{\bullet-}$ (to form the red-absorbing $\mathrm{FADH}^{\bullet}$ ) and by reduction of its partner $\mathrm{Y}_{373^{\circ}}$ by even trace amounts of residual reducing agents. All these factors are congruent with the unique properties of CraCRY in vivo.

\section{ACKNOWLEDGEMENTS}

This work obtained financial support by the International Max Planck research school for Environmental, Cellular and Molecular Biology (SF), the Deutsche Forschungsgemeinschaft (LOE: FOR 1261-2, ES 152/121), the Air Force Office of Scientific Research (AFOSR; grant No FA9550-14-1-0409) and by the French Infrastructure for Integrated Structural Biology (FRISBI: ANR-10-INBS-05).

\section{REFERENCES}

(1) Sancar, A. Structure and Function of DNA Photolyase and Cryptochrome Blue-Light Photoreceptors. Chem. Rev. 2003, 103, 2203.

(2) Lin, C. T.; Shalitin, D. Cryptochrome Structure and Signal Transduction. Annu. Rev. Plant Biol. 2003, $54,469$.

(3) Chaves, I.; Pokorny, R.; Byrdin, M.; Hoang, N.; Ritz, T.; Brettel, K.; Essen, L.-O.; van der Horst, G. T. J.; Batschauer, A.; Ahmad, M. The Cryptochromes: Blue Light Photoreceptors in Plants and Animals. Annu. Rev. Plant Biol. 2011, 62, 335.

(4) Hore, P. J.; Mouritsen, H. The Radical-Pair Mechanism of Magnetoreception. Annu. Rev. Biophys. 2016, 45, 299.

(5) Cadet, J.; Sage, E.; Douki, T. Ultraviolet Radiation-Mediated Damage to Cellular DNA. Mutation Res. 2005, 571, 3.

(6) Weber, S. Light-Driven Enzymatic Catalysis of DNA Repair: A Review of Recent Biophysical Studies on Photolyase. Biochim. Biophys. Acta Bioenerg. 2005, 1707, 1.

(7) Brettel, K.; Byrdin, M. Reaction Mechanisms of DNA Photolyase. Curr. Opin. Struct. Biol. 2010, 20, 693.

(8) Zhong, D. Electron Transfer Mechanisms of DNA Repair by Photolyase. Annu. Rev. Phys. Chem. 2015, 66, 691.

(9) Selby, C. P.; Sancar, A. A Cryptochrome/Photolyase Class of Enzymes with Single-Stranded DNASpecific Photolyase Activity. Proc. Natl. Acad. Sci. USA 2006, 103, 17696.

(10) Pokorny, R.; Klar, T.; Hennecke, U.; Carell, T.; Batschauer, A.; Essen, L. O. Recognition and Repair of UV Lesions in Loop Structures of Duplex DNA by DASH-Type Cryptochrome. Proc. Natl. Acad. Sci. USA 2008, 105, 21023. 
(11) Berrocal-Tito, G. M.; Esquivel-Naranjo, E. U.; Horwitz, B. A.; Herrera-Estrella, A. Trichoderma Atroviride PHR1, a Fungal Photolyase Responsible for DNA Repair, Autoregulates its Own Photoinduction. Eukaryotic Cell 2007, 6, 1682.

(12) Bayram, O.; Biesemann, C.; Krappmann, S.; Galland, P.; Braus, G. H. More Than a Repair Enzyme: Aspergillus nidulans Photolyase-Like CryA Is a Regulator of Sexual Development. Molec. Biol. Cell 2008, 19, 3254.

(13) Coesel, S.; Mangogna, M.; Ishikawa, T.; Heijde, M.; Rogato, A.; Finazzi, G.; Todo, T.; Bowler, C.; Falciatore, A. Diatom PtCPF1 Is a New Cryptochrome/Photolyase Family Member with DNA Repair and Transcription Regulation Activity. EMBO Rep. 2009, 10, 655.

(14) Heijde, M.; Zabulon, G.; Corellou, F.; Ishikawa, T.; Brazard, J.; Usman, A.; Plaza, P.; Martin, M. M.; Falciatore, A.; Todo, T.; Bouget, F.-Y.; Bowler, C. Characterization of Two Members of the Cryptochrome / Photolyase Family from Ostreococcus Tauri Gives Insight in the Origin and Evolution of Cryptochromes. Plant Cell Environ. 2010, 33, 1614.

(15) Kiontke, S.; Geisselbrecht, Y.; Pokorny, R.; Carell, T.; Batschauer, A.; Essen, L.-O. Crystal Structures of an Archaeal Class II DNA Photolyase and its Complex with UV-Damaged Duplex DNA. EMBO J. 2011, 30, 4437.

(16) Geisselbrecht, Y.; Fruehwirth, S.; Schroeder, C.; Pierik, A. J.; Klug, G.; Essen, L.-O. CryB from Rhodobacter Sphaeroides: A Unique Class of Cryptochromes with New Cofactors. EMBO Rep. 2012, 13, 223.

(17) Juhas, M.; von Zadow, A.; Spexard, M.; Schmidt, M.; Kottke, T.; Buechel, C. A Novel Cryptochrome in the Diatom Phaeodactylum tricornutum Influences the Regulation of Light-Harvesting Protein Levels. FEBS J. 2014, 281, 2299.

(18) Oliveri, P.; Fortunato, A. E.; Petrone, L.; Ishikawa-Fujiwara, T.; Kobayashi, Y.; Todo, T.; Antonova, O.; Arboleda, E.; Zantke, J.; Tessmar-Raible, K.; Falciatore, A. The Cryptochrome/Photolyase Family in Aquatic Organisms. Mar. Genom. 2014, 14, 23.

(19) Zhang, M.; Wang, L.; Zhong, D. Photolyase: Dynamics and Mechanisms of Repair of Sun-Induced DNA Damage. Photochem. Photobiol. 2017, 93, 78.

(20) Beel, B.; Prager, K.; Spexard, M.; Sasso, S.; Weiss, D.; Mueller, N.; Heinnickel, M.; Dewez, D.; Ikoma, D.; Grossman, A. R.; Kottke, T.; Mittag, M. A Flavin Binding Cryptochrome Photoreceptor Responds to Both Blue and Red Light in Chlamydomonas Reinhardtii. Plant Cell 2012, 24, 2992.

(21) Kottke, T.; Oldemeyer, S.; Wenzel, S.; Zou, Y.; Mittag, M. Cryptochrome Photoreceptors in Green Algae: Unexpected Versatility of Mechanisms and Functions. J. Plant Physiol. 2017, 217, 4.

(22) Zou, Y.; Wenzel, S.; Mueller, N.; Prager, K.; Jung, E.-M.; Kothe, E.; Kottke, T.; Mittag, M. An AnimalLike Cryptochrome Controls the Chlamydomonas Sexual Cycle. Plant Physiol. 2017, 174, 1334.

(23) Spexard, M.; Thoeing, C.; Beel, B.; Mittag, M.; Kottke, T. Response of the Sensory Animal-Like Cryptochrome aCRY to Blue and Red Light as Revealed by Infrared Difference Spectroscopy. Biochemistry 2014, 53, 1041.

(24) Oldemeyer, S.; Franz, S.; Wenzel, S.; Essen, L. O.; Mittag, M.; Kottke, T. Essential Role of an Unusually Long-Lived Tyrosyl Radical in the Response to Red Light of the Animal-Like Cryptochrome aCRY. J. Biol. Chem. 2016, 291, 14062.

(25) Essen, L. O. Photolyases and Cryptochromes: Common Mechanisms of DNA Repair and LightDriven Signaling? Curr. Opin. Struct. Biol. 2006, 16, 51.

(26) Heelis, P. F.; Okamura, T.; Sancar, A. Excited-State Properties of Escherichia Coli DNA Photolyase in the Picosecond to Millisecond Time Scale. Biochemistry 1990, 29, 5694.

(27) Engelhard, C.; Wang, X.; Robles, D.; Moldt, J.; Essen, L.-O.; Batschauer, A.; Bittl, R.; Ahmad, M. Cellular Metabolites Enhance the Light Sensitivity of Arabidopsis Cryptochrome through Alternate Electron Transfer Pathways. Plant Cell 2014, 26, 4519. 
(28) Ignatz, E.; Geisselbrecht, Y.; Kiontke, S.; Essen, L. O. Nicotinamide Adenine Dinucleotides Arrest Photoreduction of Class II DNA Photolyases in FADH• State. Photochem. Photobiol. 2018, 94, 81.

(29) Nohr, D.; Franz, S.; Rodriguez, R.; Paulus, B.; Essen, L. O.; Weber, S.; Schleicher, E. Extended Electron-Transfer in Animal Cryptochromes Mediated by a Tetrad of Aromatic Amino Acids. Biophys. J. 2016, 111, 301.

(30) Müller, P.; Yamamoto, J.; Martin, R.; Iwai, S.; Brettel, K. Discovery and Functional Analysis of a 4th Electron-Transferring Tryptophan Conserved Exclusively in Animal Cryptochromes and (6-4) Photolyases. Chem. Commun. 2015, 51, 15502.

(31) Martin, R.; Lacombat, F.; Espagne, A.; Dozova, N.; Plaza, P.; Yamamoto, J.; Müller, P.; Brettel, K.; de la Lande, A. Ultrafast Flavin Photoreduction in an Oxidized Animal (6-4) Photolyase through an Unconventional Tryptophan Tetrad. Phys. Chem. Chem. Phys. 2017, 19, 24493.

(32) Yamamoto, J.; Shimizu, K.; Kanda, T.; Hosokawa, Y.; Iwai, S.; Plaza, P.; Muller, P. Loss of Fourth Electron-Transferring Tryptophan in Animal (6-4) Photolyase Impairs DNA Repair Activity in Bacterial Cells. Biochemistry 2017, 56, 5356.

(33) Aubert, C.; Vos, M. H.; Mathis, P.; Eker, A. P. M.; Brettel, K. Intraprotein Radical Transfer During Photoactivation of DNA Photolyase. Nature 2000, 405, 586.

(34) Wang, H. Y.; Saxena, C.; Quan, D. H.; Sancar, A.; Zhong, D. P. Femtosecond Dynamics of Flavin Cofactor in DNA Photolyase: Radical Reduction, Local Solvation, and Charge Recombination. J. Phys. Chem. B 2005, 109, 1329.

(35) Kao, Y. T.; Tan, C.; Song, S. H.; Ozturk, N.; Li, J.; Wang, L. J.; Sancar, A.; Zhong, D. P. Ultrafast Dynamics and Anionic Active States of the Flavin Cofactor in Cryptochrome and Photolyase. J. Am. Chem. Soc. 2008, 130, 7695.

(36) Byrdin, M.; Lukacs, A.; Thiagarajan, V.; Eker, A. P. M.; Brettel, K.; Vos, M. H. Quantum Yield Measurements of Short-Lived Photoactivation Intermediates in DNA Photolyase: Toward a Detailed Understanding of the Triple Tryptophan Electron Transfer Chain. J. Phys. Chem. A 2010, $114,3207$.

(37) Liu, Z. Y.; Tan, C.; Guo, X. M.; Li, J.; Wang, L. J.; Sancar, A.; Zhong, D. P. Determining Complete Electron Flow in the Cofactor Photoreduction of Oxidized Photolyase. Proc. Natl. Acad. Sci. USA 2013, 110, 12966.

(38) Liu, Z. Y.; Tan, C.; Guo, X. M.; Li, J.; Wang, L. J.; Zhong, D. P. Dynamic Determination of Active-Site Reactivity in Semiquinone Photolyase by the Cofactor Photoreduction. J. Phys. Chem. Lett. 2014, $5,820$.

(39) Brazard, J.; Usman, A.; Lacombat, F.; Ley, C.; Martin, M. M.; Plaza, P.; Mony, L.; Heijde, M.; Zabulon, G.; Bowler, C. Spectro-Temporal Characterization of the Photoactivation Mechanism of Two New Oxidized Cryptochrome/Photolyase Photoreceptors. J. Am. Chem. Soc. 2010, 132, 4935.

(40) Lacombat, F.; Espagne, A.; Dozova, N.; Plaza, P.; Ignatz, E.; Kiontke, S.; Essen, L.-O. Femtosecond Transient Absorption Study of the Primary Photoreduction Events of a Class II Photolyase. Phys. Chem. Chem. Phys. 2018, 20, 25446.

(41) Immeln, D.; Weigel, A.; Kottke, T.; Perez Lustres, J. L. Primary Events in the Blue Light Sensor Plant Cryptochrome: Intraprotein Electron and Proton Transfer Revealed by Femtosecond Spectroscopy. J. Am. Chem. Soc. 2012, 134, 12536.

(42) Müller, P.; Bouly, J.-P.; Hitomi, K.; Balland, V.; Getzoff, E. D.; Ritz, T.; Brettel, K. ATP Binding Turns Plant Cryptochrome into an Efficient Natural Photoswitch. Sci. Rep. 2014, 4, 5175.

(43) Aubert, C.; Mathis, P.; Eker, A. P. M.; Brettel, K. Intraprotein Electron Transfer between Tyrosine and Tryptophan in DNA Photolyase from Anacystis nidulans. Proc. Natl. Acad. Sci. USA 1999, 96, 5423.

(44) Giovani, B.; Byrdin, M.; Ahmad, M.; Brettel, K. Light-Induced Electron Transfer in a Cryptochrome Blue-Light Photoreceptor. Nat. Struc. Biol. 2003, 10, 489. 
(45) Müller, P.; Ignatz, E.; Kiontke, S.; Brettel, K.; Essen, L. O. Sub-Nanosecond Tryptophan Radical Deprotonation Mediated by a Protein-Bound Water Cluster in Class II DNA Photolyases. Chem. Sci. 2018, 9, 1200.

(46) Stubbe, J.; van der Donk, W. A. Protein Radicals in Enzyme Catalysis. Chem. Rev. 1998, 98, 705.

(47) Barry, B. A.; Babcock, G. T. Tyrosine Radicals Are Involved in the Photosynthetic Oxygen-Evolving System. Proc. Natl. Acad. Sci. USA 1987, 84, 7099.

(48) Tommos, C.; Babcock, G. T. Proton and Hydrogen Currents in Photosynthetic Water Oxidation. Biochim. Biophys. Acta Bioenerg. 2000, 1458, 199.

(49) Sjödin, M.; Styring, S.; Åkermark, B.; Sun, L. C.; Hammarström, L. Proton-Coupled Electron Transfer from Tyrosine in a Tyrosine-Ruthenium-Tris-Bipyridine Complex: Comparison with Tyrosine ${ }_{z}$ Oxidation in Photosystem II. J. Am. Chem. Soc. 2000, 122, 3932.

(50) Rappaport, F.; Boussac, A.; Force, D. A.; Peloquin, J.; Brynda, M.; Sugiura, M.; Un, S.; Britt, R. D.; Diner, B. A. Probing the Coupling between Proton and Electron Transfer in Photosystem II Core Complexes Containing a 3-Fluorotyrosine. J. Am. Chem. Soc. 2009, 131, 4425.

(51) Hammarström, L.; Styring, S. Proton-Coupled Electron Transfer of Tyrosines in Photosystem II and Model Systems for Artificial Photosynthesis: The Role of a Redox-Active Link between Catalyst and Photosensitizer. Energy \& Environmental Science 2011, 4, 2379.

(52) Barry, B. A. Proton Coupled Electron Transfer and Redox Active Tyrosines in Photosystem II. Journal of Photochemistry and Photobiology B-Biology 2011, 104, 60.

(53) Schlodder, E.; Cetin, M.; Lendzian, F. Temperature Dependence of the Oxidation Kinetics of Tyr(Z) and $\operatorname{Tyr}(\mathrm{D})$ in Oxygen-Evolving Photosystem II Complexes Throughout the Range from $320 \mathrm{~K}$ to $5 \mathrm{~K}$. Biochim. Biophys. Acta Bioenerg. 2015, 1847, 1283.

(54) Tommos, C.; Skalicky, J. J.; Pilloud, D. L.; Wand, A. J.; Dutton, P. L. De Novo Proteins as Models of Radical Enzymes. Biochemistry 1999, 38, 9495.

(55) Rappaport, F.; Diner, B. A. Primary Photochemistry and Energetics Leading to the Oxidation of the $(\mathrm{Mn}) 4 \mathrm{ca}$ Cluster and to the Evolution of Molecular Oxygen in Photosystem II. Coord. Chem. Rev. 2008, 252, 259.

(56) van Best, J. A.; Mathis, P. Kinetics of Reduction of the Oxidized Primary Electron Donor of Photosystem II in Spinach Chloroplasts and in Chlorella Cells in the Microsecond and Nanosecond Time Ranges Following Flash Excitation. Biochim Biophys Acta 1978, 503, 178.

(57) Brettel, K.; Schlodder, E.; Witt, H. T. Nanosecond Reduction Kinetics of Photooxidized Chlorophyll$\mathrm{a}_{\| 1}(\mathrm{P}-680)$ in Single Flashes as a Probe for the Electron Pathway, $\mathrm{H}^{+}$-Release and Charge Accumulation in the $\mathrm{O}_{2}$-Evolving Complex. Biochim. Biophys. Acta Bioenerg. 1984, 766, 403.

(58) Gerken, S.; Brettel, K.; Schlodder, E.; Witt, H. T. Optical Characterization of the Immediate Electron Donor to Chlorophyll $\mathrm{A}_{\pi}{ }^{+}$in $\mathrm{O}_{2}$-Evolving Photosystem II Complexes: Tyrosine as Possible Electron Carrier between Chlorophyll $\mathrm{A}_{\pi}$ and the Water-Oxidizing Manganese Complex. FEBS Lett. 1988, 237, 69.

(59) Styring, S.; Sjöholm, J.; Mamedov, F. Two Tyrosines That Changed the World: Interfacing the Oxidizing Power of Photochemistry to Water Splitting in Photosystem II. Biochim. Biophys. Acta Bioenerg. 2012, 1817, 76.

(60) Dixon, W. T.; Murphy, D. Determination of Acidity Constants of Some Phenol Radical Cations by Means of Electron-Spin Resonance. J. Chem. Soc. Faraday Trans. I/ 1976, 72, 1221.

(61) Gauden, M.; van Stokkum, I. H. M.; Key, J. M.; Luhrs, D. C.; Van Grondelle, R.; Hegemann, P.; Kennis, J. T. M. Hydrogen-Bond Switching through a Radical Pair Mechanism in a Flavin-Binding Photoreceptor. Proc. Natl. Acad. Sci. USA 2006, 103, 10895.

(62) Mathes, T.; van Stokkum, I. H. M.; Stierl, M.; Kennis, J. T. M. Redox Modulation of Flavin and Tyrosine Determines Photoinduced Proton-Coupled Electron Transfer and Photoactivation of BLUF Photoreceptors. J. Biol. Chem. 2012, 287, 31725. 
(63) Mathes, T.; Zhu, J. Y.; van Stokkum, I. H. M.; Groot, M. L.; Hegemann, P.; Kennis, J. T. M. Hydrogen Bond Switching among Flavin and Amino Acids Determines the Nature of Proton-Coupled Electron Transfer in BLUF Photoreceptors. J. Phys. Chem. Lett. 2012, 3, 203.

(64) Hammes-Schiffer, S.; Stuchebrukhov, A. A. Theory of Coupled Electron and Proton Transfer Reactions. Chem. Rev. 2010, 110, 6939.

(65) Weinberg, D. R.; Gagliardi, C. J.; Hull, J. F.; Murphy, C. F.; Kent, C. A.; Westlake, B. C.; Paul, A.; Ess, D. H.; McCafferty, D. G.; Meyer, T. J. Proton-Coupled Electron Transfer. Chem. Rev. 2012, 112, 4016.

(66) Saveant, J. M. Electrochemical Approach to Proton-Coupled Electron Transfers: Recent Advances. Energy \& Environmental Science 2012, 5, 7718.

(67) Migliore, A.; Polizzi, N. F.; Therien, M. J.; Beratan, D. N. Biochemistry and Theory of ProtonCoupled Electron Transfer. Chem. Rev. 2014, 114, 3381.

(68) Gentry, E. C.; Knowles, R. R. Synthetic Applications of Proton-Coupled Electron Transfer. Acc. Chem. Res. 2016, 49, 1546.

(69) Odella, E.; Mora, S. J.; Wadsworth, B. L.; Huynh, M. T.; Goings, J. J.; Liddell, P. A.; Groy, T. L.; Gervaldo, M.; Sereno, L. E.; Gust, D.; Moore, T. A.; Moore, G. F.; Hammes-Schiffer, S.; Moore, A. L. Controlling Proton-Coupled Electron Transfer in Bioinspired Artificial Photosynthetic Relays. J Am Chem Soc 2018, 140, 15450.

(70) Franz, S.; Ignatz, E.; Wenzel, S.; Zielosko, H.; Putu, E. P. G. N.; Maestre-Reyna, M.; Tsai, M.-D.; Yamamoto, J.; Mittag, M.; Essen, L.-O. Structure of the Bifunctional Cryptochrome aCRY from Chlamydomonas Reinhardtii. Nucleic Acids Res. 2018, 46, 8010.

(71) Byrdin, M.; Villette, S.; Espagne, A.; Eker, A. P. M.; Brettel, K. Polarized Transient Absorption to Resolve Electron Transfer between Tryptophans in DNA Photolyase. J. Phys. Chem. B 2008, 112, 6866.

(72) Yoshimura, A.; Hoffman, M. Z.; Sun, H. An Evaluation of the Excited-State Absorption-Spectrum of $\mathrm{Ru}(\mathrm{Bpy})_{3}{ }^{2+}$ in Aqueous and Acetonitrile Solutions. J. Photochem. Photobiol. A-Chem. 1993, 70, 29.

(73) Ekvall, K.; van der Meulen, P.; Dhollande, C.; Berg, L. E.; Pommeret, S.; Naskrecki, R.; Mialocq, J. C. Cross Phase Modulation Artifact in Liquid Phase Transient Absorption Spectroscopy. J. Appl. Phys. 2000, 87, 2340.

(74) van Stokkum, I. H. M.; Delmar, S. L.; van Grondelle, R. Global and Target Analysis of Time-Resolved Spectra. Biochim. Biophys. Acta Bioenerg. 2004, 1657, 82.

(75) Byrdin, M.; Thiagarajan, V.; Villette, S.; Espagne, A.; Brettel, K. Use of Ruthenium Dyes for Subnanosecond Detector Fidelity Testing in Real Time Transient Absorption. Rev. Sci. Instr. 2009, $80,043102$.

(76) Müller, P.; Brettel, K.; Grama, L.; Nyitrai, M.; Lukacs, A. Photochemistry of Wild-Type and N378D Mutant E. coli DNA Photolyase with Oxidized FAD Cofactor Studied by Transient Absorption Spectroscopy. ChemPhysChem 2016, 17, 1329.

(77) Müller, P.; Brettel, K. [Ru(Bpy $\left.)_{3}\right]^{2+}$ as a Reference in Transient Absorption Spectroscopy: Differential Absorption Coefficients for Formation of the Long-Lived 3MLCT Excited State. Photochem. Photobiol. Sci. 2012, 11, 632.

(78) Solar, S.; Getoff, N.; Surdhar, P. S.; Armstrong, D. A.; Singh, A. Oxidation of Tryptophan and Nmethylindole by $\mathrm{N}_{3}{ }^{\circ}, \mathrm{Br}_{2}{ }^{\circ-}$, and $(\mathrm{SCN})_{2}{ }^{\cdot-}$ Radicals in Light-Water and Heavy-Water Solutions: A Pulse Radiolysis Study. J. Phys. Chem. 1991, 95, 3639.

(79) Massey, V.; Palmer, G. On Existence of Spectrally Distinct Classes of Flavoprotein Semiquinones . A New Method for Quantitative Production of Flavoprotein Semiquinones. Biochemistry 1966, 5, 3181. 
Biol. Chem. 2007, 282, 13011.

(81) Nag, L.; Sournia, P.; Myllykallio, H.; Liebl, U.; Vos, M. H. Identification of the $\mathrm{TyrOH}^{{ }^{+}}$Radical Cation in the Flavoenzyme TrmFO. J. Am. Chem. Soc. 2017, 139, 11500.

(82) Meyer, B.; Schlodder, E.; Dekker, J. P.; Witt, H. T. O ${ }_{2}$ Evolution and Chl $\mathrm{a}^{+}{ }^{\prime \prime}\left(\mathrm{P}-680^{+}\right)$Nanosecond Reduction Kinetics in Single Flashes as a Function of pH. Biochim. Biophys. Acta 1989, 974, 36.

(83) Karge, M.; Irrgang, K. D.; Sellin, S.; Feinaugle, R.; Liu, B.; Eckert, H. J.; Eichler, H. J.; Renger, G. Effects of Hydrogen Deuterium Exchange on Photosynthetic Water Cleavage in Ps II Core Complexes from Spinach. FEBS Lett. 1996, 378, 140.

(84) Haumann, M.; Bogershausen, O.; Cherepanov, D.; Ahlbrink, R.; Junge, W. Photosynthetic Oxygen Evolution: H/D Isotope Effects and the Coupling between Electron and Proton Transfer During the Redox Reactions at the Oxidizing Side of Photosystem II. Photosynth. Res. 1997, 51, 193.

(85) Schilstra, M. J.; Rappaport, F.; Nugent, J. H. A.; Barnett, C. J.; Klug, D. R. Proton/Hydrogen Transfer Affects the S-state-Dependent Microsecond Phases of P680+ Reduction During Water Splitting. Biochemistry 1998, 37, 3974.

(86) Liu, T.; Guo, M.; Orthaber, A.; Lomoth, R.; Lundberg, M.; Ott, S.; Hammarström, L. Accelerating Proton-Coupled Electron Transfer of Metal Hydrides in Catalyst Model Reactions. Nat. Chem. 2018, 10, 881.

(87) Grimsley, G. R.; Scholtz, J. M.; Pace, C. N. A Summary of the Measured pK Values of the lonizable Groups in Folded Proteins. Protein Sci. 2009, 18, 247.

(88) Hazra, A.; Soudackov, A. V.; Hammes-Schiffer, S. Isotope Effects on the Nonequilibrium Dynamics of Ultrafast Photoinduced Proton-Coupled Electron Trasfer Reactions in Solution. J. Phys. Chem. Lett. 2011, 2, 36.

(89) Page, C. C.; Moser, C. C.; Chen, X. X.; Dutton, P. L. Natural Engineering Principles of Electron Tunnelling in Biological Oxidation-Reduction. Nature 1999, 402, 47.

(90) Kim, J.; Heo, W.; Joo, T. Excited State Intramolecular Proton Transfer Dynamics of 1-Hydroxy-2Acetonaphthone. J. Phys. Chem. B 2015, 119, 2620.

(91) Chattoraj, M.; King, B. A.; Bublitz, G. U.; Boxer, S. G. Ultra-Fast Excited State Dynamics in Green Fluorescent Protein: Multiple States and Proton Transfer. Proc. Natl. Acad. Sci. USA 1996, 93, 8362.

(92) Shinobu, A.; Agmon, N. Proton Wire Dynamics in the Green Fluorescent Protein. J Chem Theory Comput 2017, 13, 353.

(93) Krishtalik, L. I. The Mechanism of the Proton Transfer: An Outline. Biochim. Biophys. Acta Bioenerg. 2000, 1458, 6.

(94) Umena, Y.; Kawakami, K.; Shen, J. R.; Kamiya, N. Crystal Structure of Oxygen-Evolving Photosystem II at a Resolution of $1.9 \AA$ A Nature 2011, 473, 55.

(95) Conjeaud, H.; Mathis, P. The Effect of $\mathrm{pH}$ on the Reduction Kinetics of P-680 in Tris-Treated Chloroplasts. Biochim. Biophys. Acta 1980, 590, 353.

(96) Schlodder, E.; Meyer, B. pH Dependence of Oxygen Evolution and Reduction Kinetics of Photooxidized Chlorophyll all (P-680) in Photosystem II Particles from Synechococcus Sp. Biochim. Biophys. Acta 1987, 890, 23.

(97) Ahlbrink, R.; Haumann, M.; Cherepanov, D.; Bogershausen, O.; Mulkidjanian, A.; Junge, W. Function of Tyrosine Z in Water Oxidation by Photosystem II: Electrostatical Promotor Instead of Hydrogen Abstractor. Biochemistry 1998, 37, 1131.

(98) Diner, B. A.; Force, D. A.; Randall, D. W.; Britt, R. D. Hydrogen Bonding, Solvent Exchange, and Coupled Proton and Electron Transfer in the Oxidation and Reduction of Redox-Active Tyrosine $Y_{z}$ in Mn-Depleted Core Complexes of Photosystem II. Biochemistry 1998, 37, 17931. 
(99) Hays, A. M. A.; Vassiliev, I. R.; Golbeck, J. H.; Debus, R. J. Role of D1-His190 in the Proton-Coupled Oxidation of Tyrosine $Y_{z}$ in Manganese-Depleted Photosystem II. Biochemistry 1999, 38, 11851.

(100) Saito, K.; Shen, J. R.; Ishida, T.; Ishikita, H. Short Hydrogen Bond between Redox-Active Tyrosine $Y_{z}$ and D1-His190 in the Photosystem II Crystal Structure. Biochemistry 2011, $50,9836$.

(101) Udvarhelyi, A.; Domratcheva, T. Photoreaction in BLUF Receptors: Proton-Coupled Electron Transfer in the Flavin-Gln-Tyr System. Photochem. Photobiol. 2011, 87, 554.

(102) Sayfutyarova, E. R.; Goings, J. J.; Hammes-Schiffer, S. Electron-Coupled Double Proton Transfer in the SIr1694 BLUF Photoreceptor: A Multireference Electronic Structure Study. J. Phys. Chem. B 2019, 123, 439.

(103) Heelis, P. F. The Photophysical and Photochemical Properties of Flavins (Isoalloxazines). Chem. Soc. Rev. 1982, 11, 15.

(104) Beel, B.; Muller, N.; Kottke, T.; Mittag, M. News About Cryptochrome Photoreceptors in Algae. Plant Signal. Behav. 2013, 8, e22870. 


\section{FIGURES}
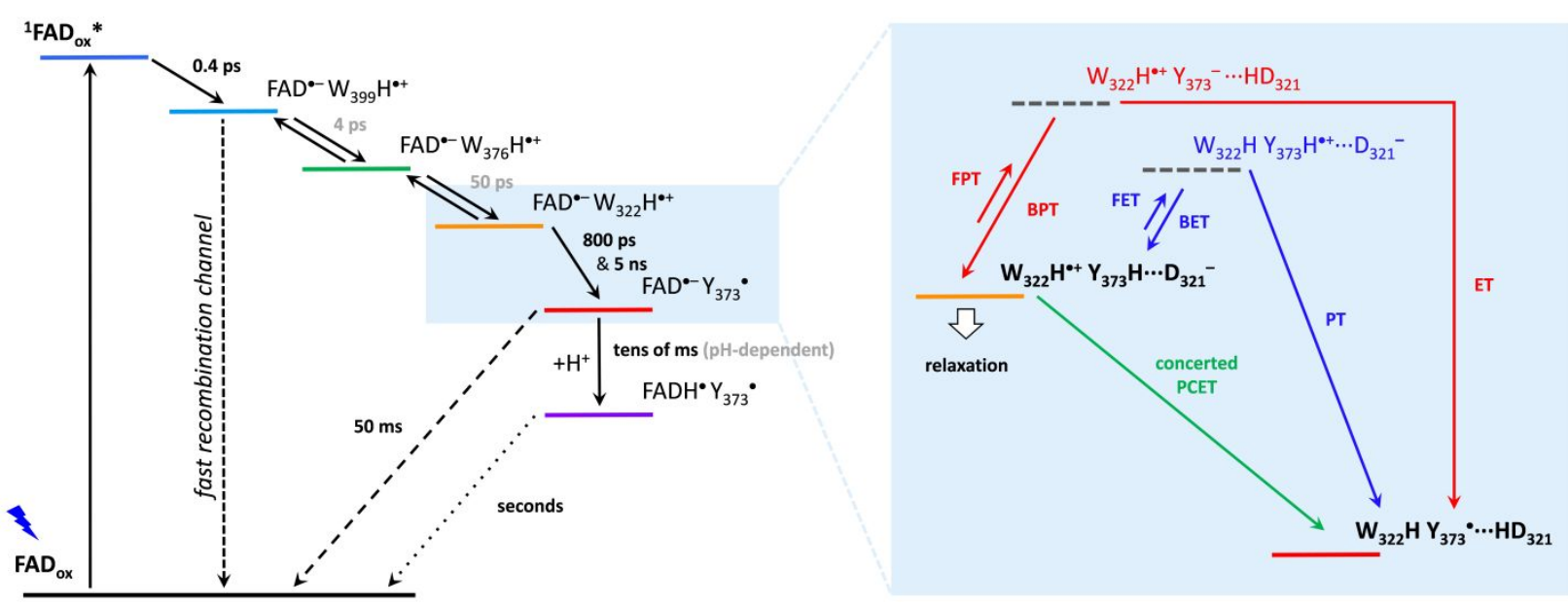

Scheme 1. Tentative mechanism of $F A D_{\text {ox }}$ photoreduction in CraCRY. Possible pathways leading to $\mathrm{Y}_{373}$ oxidation by $\mathrm{W}_{322} \mathrm{H}^{\bullet+}$ (light-blue background) are shown in the right part of the scheme (see Section 4.2.2 for detailed discussion).
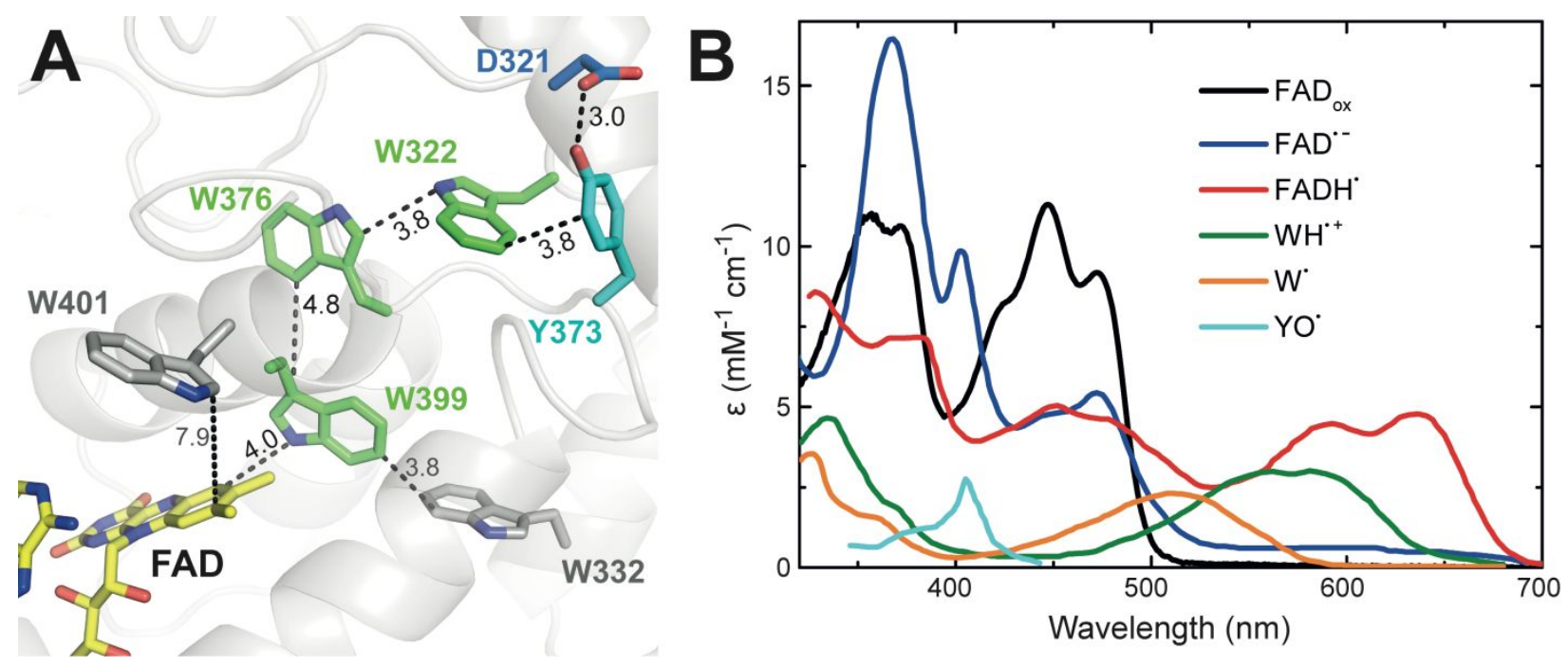

Figure 1. (A) Local structure of CraCRY in the flavin photoreduction site (PDB entry $5 Z \mathrm{ZM0} 0^{70}$; resolution 1.6 ̊). FAD is represented in yellow, the conserved tryptophan triad in green, $Y_{373}$ in cyan, D321 in blue and the side tryptophans as reported by Li et al. ${ }^{37}$ in grey. Closest edge-to-edge distances are indicated in $\AA$. (B) Reference species spectra used for spectral analysis (see SI, Section S5.1). 

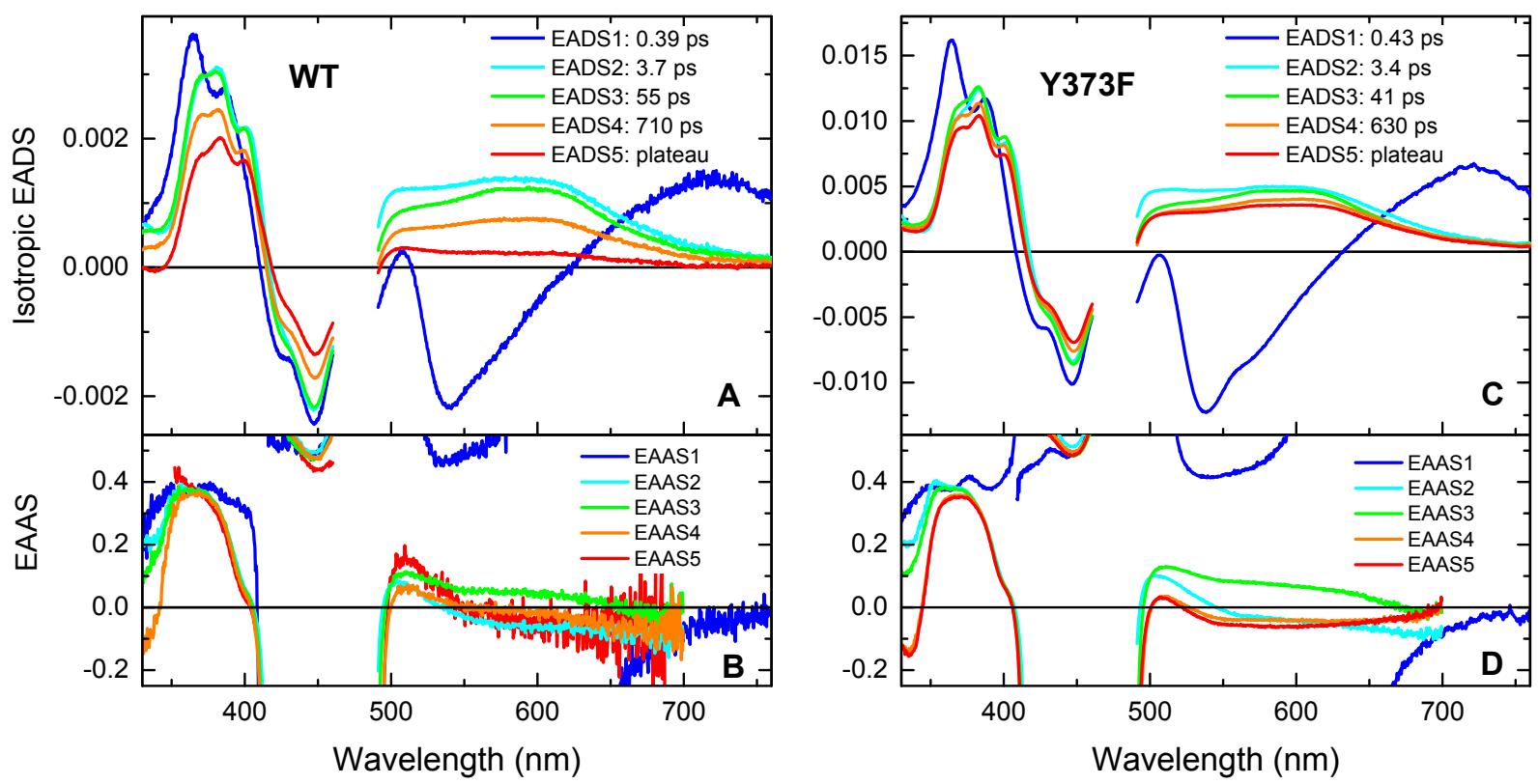

Figure 2. Top: Isotropic EADS deduced from the global analysis of the polarized transient absorption spectra of $\mathrm{CraCRY}$ in $\mathrm{H}_{2} \mathrm{O}$ buffer (A: WT; C: Y373F) with a sum of 4 exponentials and a plateau. Bottom: Corresponding EAAS (B: WT; D: Y373F). Exceedingly noisy parts of these spectra above $700 \mathrm{~nm}$ or below $350 \mathrm{~nm}$ have been masked.

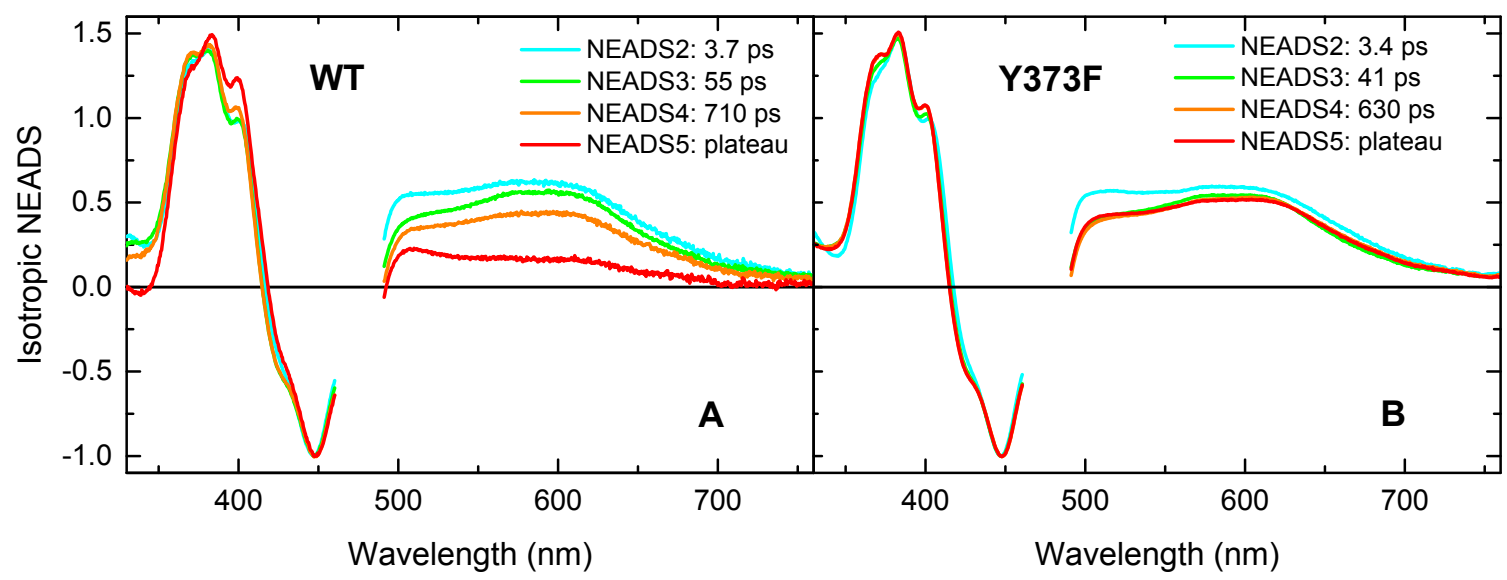

Figure 3. Normalized isotropic EADS of WT (A) and Y373F (B) in $\mathrm{H}_{2} \mathrm{O}$ buffer, after decay of the initial excited state (EADS1 excluded). Normalization was made at the maximum of the bleaching band, at 447 $\mathrm{nm}$. 


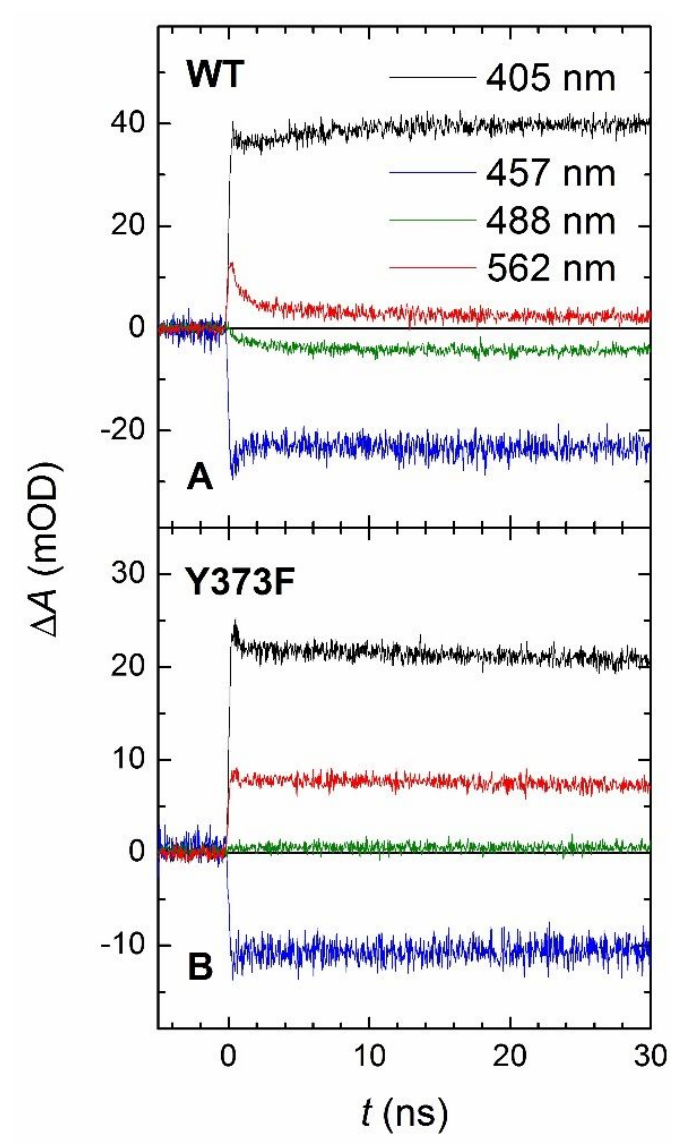

Figure 4. Flash-induced absorption changes on a ns timescale for $(A) \sim 110 \mu \mathrm{M}$ WT and (B) $\sim 60 \mu \mathrm{M} Y 373 \mathrm{~F}$ mutant CraCRY at four characteristic wavelengths (see Figures S2A and S3A for signals at additional wavelengths). The samples were excited at $355 \mathrm{~nm}$ by $100 \mathrm{ps}$ pulses of an energy of $\sim 2 \mathrm{~mJ}$ per $\mathrm{cm}^{2}$. Individual traces in both panels are averages of 64 flashes recorded with a repetition rate of $2 \mathrm{~Hz}$. 

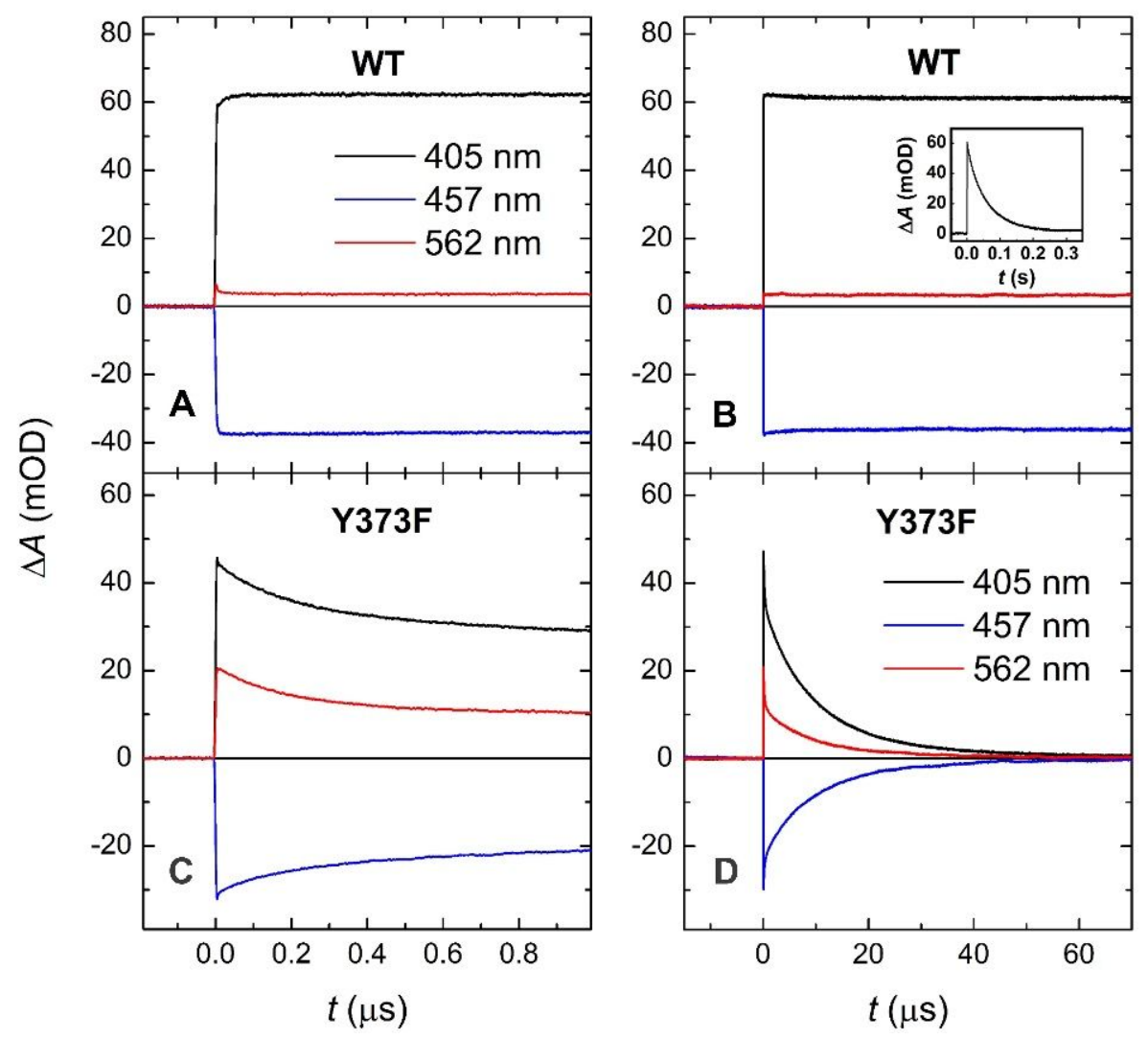

Figure 5. Flash-induced absorption changes on sub- $\mu$ s to $\mu$ s timescales for $~ 60 \mu M$ WT (A \& B) and Y373F mutant (C \& D) CraCRY at three characteristic wavelengths (see Figure S3B and C for additional wavelengths). The samples were excited at $475 \mathrm{~nm}$ by $5 \mathrm{~ns}$ pulses of an energy of $\sim 5 \mathrm{~mJ}$ per $\mathrm{cm}^{2}(\sim 4 \mathrm{~mJ}$ for the Y373F mutant). Individual traces in panels A and B (WT) are averages of four single flash signals spaced by $\sim 1$ minute, those in panels $C$ and $D$ (Y373F) are averages of 16 flashes recorded with a repetition rate of $2 \mathrm{~Hz}$. The trace in the inset of panel $B$ is a single-flash signal recorded at $405 \mathrm{~nm}$.
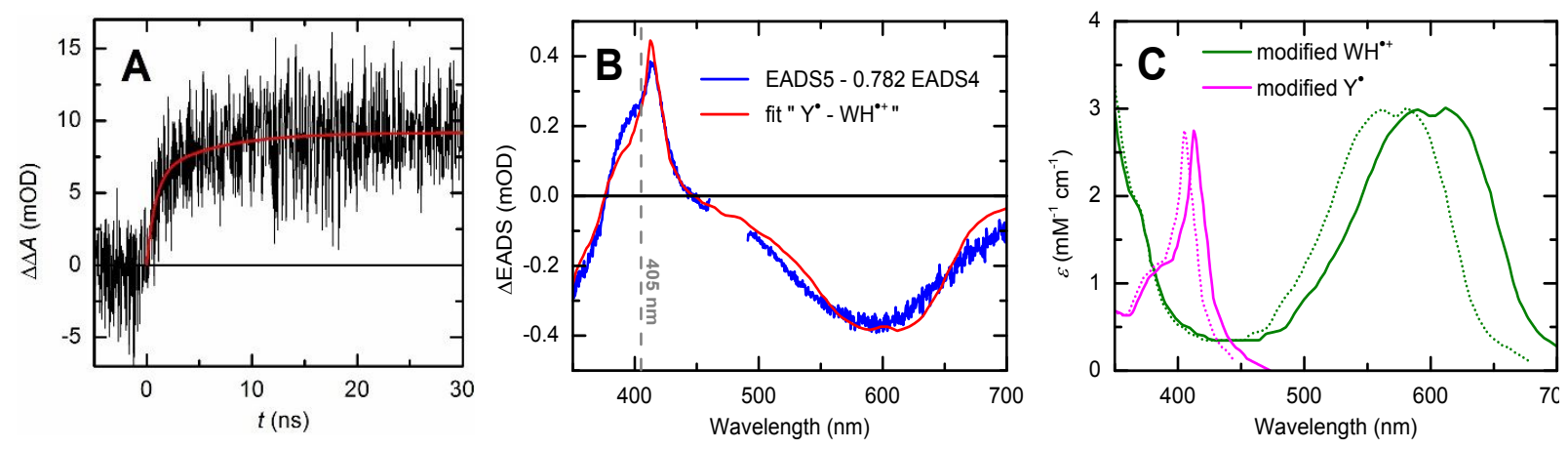

Figure 6. (A) Black: signal constructed by subtraction of the inverted and normalized $457 \mathrm{~nm}$ signal in Figure 4A from the signal at $405 \mathrm{~nm}$ (ibid.). The constructed signal should reflect the crude kinetics of $\mathrm{Y}^{\bullet}$ formation by "correcting" the $405 \mathrm{~nm} \Delta A$ signal for contributions from FAD`-; red: bi-exponential fit of 
the black signal. (B) Spectral fit of the EADS5 - $\phi$ EADS4 difference (WT in $\mathrm{H}_{2} \mathrm{O}$ buffer), according to Equation S5 (SI, Section S5.3). The best fit (red) yields $\phi=0.782$. (C) Corresponding modified $\mathrm{Y}^{\bullet}$ and $\mathrm{WH}^{\bullet+}$ spectra (original spectra are recalled in dotted lines). 


\section{TABLES}

Table 1. Time constants of the global multiexponential fit of the polarized transient absorption spectra of CraCRY, WT in $\mathrm{H}_{2} \mathrm{O}$ and $\mathrm{D}_{2} \mathrm{O}$ buffer, and $\mathrm{Y} 373 \mathrm{~F}$ in $\mathrm{H}_{2} \mathrm{O}$ buffer. Fit errors (95\% confidence interval) are indicated after the \pm sign. The coefficient of determination $\left(R^{2}\right)$ is given in the last column.

\begin{tabular}{lcccccc}
\hline protein & buffer & $\tau_{1}(\mathrm{ps})$ & $\tau_{2}(\mathrm{ps})$ & $\tau_{3}(\mathrm{ps})$ & $\tau_{4}(\mathrm{ps})$ & $\mathrm{R}^{2}$ \\
\hline \multirow{2}{*}{ WT } & $\mathrm{H}_{2} \mathrm{O}$ & $0.39 \pm 0.02$ & $3.7 \pm 0.8$ & $55 \pm 6$ & $710 \pm 80$ & 0.999468 \\
& $\mathrm{D}_{2} \mathrm{O}$ & $0.37 \pm 0.02$ & $4.9 \pm 1.0$ & $56 \pm 9$ & $600 \pm 70$ & 0.999278 \\
\hline $\mathrm{Y} 373 \mathrm{~F}$ & $\mathrm{H}_{2} \mathrm{O}$ & $0.43 \pm 0.01$ & $3.4 \pm 0.4$ & $41 \pm 4$ & $630 \pm 110$ & 0.999904 \\
\hline
\end{tabular}

Table 2. Evolution-associated anisotropies taken at $610 \mathrm{~nm}\left(r_{\mathrm{i}}=\mathrm{EAAS}_{\mathrm{i}}(610 \mathrm{~nm})\right.$, averaged over $\left.5 \mathrm{~nm}\right)$, after initial decay of the excited state.

\begin{tabular}{lccccc}
\hline protein & buffer & $r_{2}$ & $r_{3}$ & $r_{4}$ & $r_{5}$ \\
\hline \multirow{2}{*}{ WT } & $\mathrm{H}_{2} \mathrm{O}$ & -0.062 & 0.041 & -0.017 & -0.053 \\
& $\mathrm{D}_{2} \mathrm{O}$ & -0.062 & 0.071 & -0.027 & -0.101 \\
\hline $\mathrm{Y373F}$ & $\mathrm{H}_{2} \mathrm{O}$ & -0.047 & 0.063 & -0.042 & -0.059 \\
\hline
\end{tabular}

Table 3. Expected intrinsic anisotropies attached to the different $\mathrm{WH}^{\bullet+}$ radicals of the photoreduction site, according to the crystal structure of WT-CraCRY (PDB entry 5ZM0). ${ }^{70}$ Errors indicated after the \pm sign correspond to variations of $\pm 5^{\circ}$ of the directions of both $\mathrm{FAD}_{\mathrm{ox}}$ and $\mathrm{WH}^{\bullet+}$ transition moments within the molecular plane. The bottom line proposes at tentative correction of these values, including a contribution from $F A D^{\bullet-}$ (see text).

\begin{tabular}{lccc||cc}
\hline & $W_{399}$ & $W_{376}$ & $W_{322}$ & $W_{401}$ & $W_{332}$ \\
\hline raw & $0.004 \pm 0.07$ & $0.27 \pm 0.05$ & $-0.06 \pm 0.07$ & $-0.20 \pm 0.02$ & $0.20 \pm 0.07$ \\
\hline correction & $-0.02 \pm 0.07$ & $0.20 \pm 0.05$ & $-0.07 \pm 0.07$ & $-0.18 \pm 0.02$ & $0.14 \pm 0.07$ \\
\hline
\end{tabular}




\section{ASSOCIATED CONTENT}

Supporting Information. S1) Steady-state absorption spectroscopy, S2) Real-time transient absorption spectroscopy, S3) Femtosecond TA and anisotropy spectra, S4) Global kinetic analysis of fs TAS data, S5) Spectral fitting of fs TAS spectra.

\section{Corresponding Authors}

*Pascal Plaza: Email: pascal.plaza@ens.fr. Phone: +33 144322414.

*Pavel Müller: Email: pavel.muller@i2bc.paris-saclay.fr. Phone: +33 169089014.

*Lars-Oliver Essen: Email: essen@chemie.uni-marburg.de. Phone: +49 64212822032.

\section{Conflicts of interest}

There are no conflicts to declare. 